CPHT RR 002.0104

LPT-ORSAY 04-11

hep-th/0401201

\title{
Non-tachyonic Scherk-Schwarz compactifications, cosmology and moduli stabilization
}

\author{
E. Dudas ${ }^{\dagger, \star}$ and C. Timirgaziu ${ }^{\star}$ \\ † Centre de Physique Théorique ${ }^{1}$, Ecole Polytechnique, F-91128 Palaiseau \\ * Laboratoire de Physique Théorique ${ }^{2}$ \\ Université de Paris-Sud, Bât. 210, F-91405 Orsay Cedex
}

\begin{abstract}
It is well-known that Scherk-Schwarz compactifications in string theory have a tachyon in the closed string spectrum appearing for a critical value of a compact radius. The tachyon can be removed by an appropriate orientifold projection in type II strings, giving rise to tachyon-free compactifications. We present explicit examples of this type in various dimensions, including six and four-dimensional chiral examples, with softly broken supersymmetry in the closed sector and non-BPS configurations in the open sector. These vacua are interesting frameworks for studying various cosmological issues. We discuss four-dimensional cosmological solutions and moduli stabilization triggered by nonperturbative effects like gaugino condensation on D-branes and fluxes.
\end{abstract}

\footnotetext{
${ }^{1}$ Unité mixte du CNRS et de l'EP, UMR 7644.

${ }^{2}$ Unité Mixte de Recherche du CNRS (UMR 8627).
} 


\section{Introduction}

Supersymmetry breaking in string theory is a still open and central question for string phenomenology and cosmology. At the perturbative string level there are basically three known ways of breaking supersymmetry : Scherk-Schwarz type compactifications [1], breaking induced by internal magnetic fields (T-dual to branes intersecting at angles) [2] and breaking by nonBPS configurations [3]. There are also completely nonsupersymmetric heterotic or type $\mathrm{O}$ string constructions [4, 5].

Scherk-Schwarz string compactifications have generically a tachyon-like field in their spectrum that appears for a critical value of a compact radius $R^{2}=2 \alpha^{\prime}$ [1]. Orientifolds [6] (see [7] for reviews on orientifold constructions and extensive references) of such vacua were constructed in [8] and 9]. It was then shown in [10, [11] that the lowest mass would-be tachyon field can be eliminated by using an orientifold projection similar to the one used in type $\mathrm{O}$ strings $[5]$. In two more recent papers [11, 12, we studied classical solutions of these models and found nonsingular 9D solutions in the compact space, related by a change of coordinates to the supersymmetric solution with (constant velocity) moving spacetime boundaries. A compactification to four dimensions was also performed. As a result, an expanding FRW universe was found, together with a natural way of producing three large spacetime dimensions and a much slower time evolution of the compact space.

The goal of this paper is to generalize these results to lower dimensions, construct vacua which have the necessary features for finding later on realistic examples, in particular chiral fermions and start studying nonperturbative issues like moduli stabilization. The stabilization will change the cosmological solutions into Minkowski or de Sitter solutions, depending on details of moduli stabilization.

The paper is organized as follows. Section 2 describes the basic brane-orientifold plane system appearing in all the non-tachyonic constructions of our paper and its basic features. Section 3 presents an eight dimensional example where the two-dimensional compact space is a tilted torus, and a chiral six dimensional example. Section 4 presents four-dimensional examples based on $Z_{2} \times Z_{2}$ orbifolds, including a chiral model based on a model with discrete torsion.

Section 5 represents a first excursion into the issue of (Kahler) moduli stabilization triggered by nonperturbatively induced brane potentials, like for example gaugino condensation, and/or fluxes. We show that nonperturbative dynamics can stop the moving brane and stabilize the modulus describing the distance between the boundaries and also the other volume Kahler modulus, in analogy with the phenomenological analysis performed in [13] in a different context. In an example with nonpertubative gaugino condensation, naturally realized in our string examples, stabilization is possible only with gaugino condensation combined with constant terms in 
the potential in a positive perfect square, in close analogy with the four-dimensional heterotic examples of gaugino condensation. We find also a no-scale structure for the dilaton, which is therefore not stabilized by the brane dynamics we are discussing. In order to stabilise the dilaton we could add in addition a combination of NS-NS and RR fluxes, following [14. The resulting spacetime can be Minkowski or de Sitter, depending on the parameters entering the stabilization mechanism.

The Appendix provides our conventions and useful formulae for $Z_{2} \times Z_{2}$ characters used in the text.

\section{The Dp-brane / $\overline{O p}_{-}$-plane system in string theory}

Time-dependence in string theory relies until now on some simple and computationally tractable examples [15, 16]. The best known example is the brane-antibrane pair. This system breaks completely supersymmetry and the open string spectrum contains a tachyon stretched between the brane and the antibrane, reflecting the attraction between them. The open string tachyon condensation and, more recently, the detailed time evolution triggered by the tachyon field was subject to intense study starting from the seminal papers of A.Sen [17.

An interesting but much less studied system is the Dp-brane / $\overline{O p}_{-}$-plane system. In our terminology, the $\overline{O p}_{-}$-plane is a (non-dynamical) anti-orientifold plane of $p+1$ world-volume dimensions having the same quantum numbers as a coincident superposition of $2^{p-4}$ antibranes $\overline{D p}$. The branes and the antiorientifold planes we consider attract each other, in close analogy with the brane-antibrane system. The orientifold planes are non-dynamical objects, however, and this implies that they cannot annihilate with the branes. This reflects, in particular, in the fact that there is no tachyon stretched in the system we consider. Their mutual attraction, however, clearly generates a time-dependence which is one of the main motivations for the string constructions in the present paper. The tension and RR charge of a system of $n D p$ branes and an $\overline{O p}_{-}$plane are given by

$$
\mathcal{T}=\left(n+2^{p-4}\right) \mathcal{T}_{p} \quad, \quad q=\left(n-2^{p-4}\right) \mathcal{T}_{p}
$$

where $\mathcal{T}_{p}$ is the tension of an elementary Dp brane, and are such that $\mathcal{T}>q$. Let us now place ourselves in a reference frame in which the system moves with a constant velocity $v$. In that frame, the effective tension and $\mathrm{RR}$ charge are $\left(T_{\mathrm{eff}}=T \sqrt{1-v^{2}}, q\right)$. If we observe the non-BPS system in a frame where $T_{\text {eff }}=q$, then it will look as the BPS system $\mathrm{n}$ Dp- $O p_{+}$, where the (usual) $O p_{+}$plane has the tension and RR charge $\left(-2^{p-4},-2^{p-4}\right)$. In particular it will consistently couple to the static and supersymmetric geometry corresponding to an appropriate BPS system. Therefore the system we are considering, $n D p-\overline{O p}_{-}$, moving with a constant 
velocity $v=t h \xi$, where

$$
\operatorname{ch} \xi=\frac{n+2^{p-4}}{n-2^{p-4}},
$$

behaves similarly to the BPS configuration $n D p-O p_{+}$.

The explicit string examples we provide in Sections 3 and 4 contain actually, in addition to the system mentioned above, additional branes and conventional orientifold planes $O_{+}$. A further interesting property of this class of string vacua is that the $O_{+}-\bar{O}_{-}$system, even if of orientifold-antiorientifold type, has the property of eliminating the closed string tachyon present in the string vacua before orientifolding. More precisely, as shown in the $9 \mathrm{~d}$ example constructed in [11] and in the lower dimensional examples in Section 3 and 4, $O_{+}-\bar{O}_{-}$pairs appear in Scherk-Schwarz compactifications (with a peculiar orientifold projection), which have in the closed string spectrum a would-be tachyon [1]. Standard string techniques show that $O_{+}-\overline{O p}_{-}$interaction is described by (for the definition of the characters, see the appendix)

$$
\begin{aligned}
& \text { loop channel } \quad-\left(O_{8}-C_{8}\right), \\
& \text { tree - level channel } \quad-\left(V_{8}+S_{8}\right),
\end{aligned}
$$

where, as usual, in the Klein bottle amplitudes, $(O, V)$ describe the NS-NS sector (a scalar tachyon, graviton and antisymmetric tensor), whereas $(S, C)$ describe the RR sector. These amplitudes are similar to the brane-antibrane one, but their interpretation is quite different, since they just symmetrize (or antisymmetrize) states already existent in the torus amplitude. In particular, the scalar closed string tachyon is antisymmetrized and is therefore eliminated from the spectrum.

More precisely, as shown in [11 and discussed in more detail in the compactified models in Sections 3 and 4, these exotic orientifold configurations are generated by the orientifold projection $\Omega^{\prime}=\Omega \Pi_{y}(-1)^{f_{L}}$, where $(-1)^{f_{L}}$ is the left world-sheet fermion number ${ }^{3}$ and $y$ is the coordinate used to break supersymmetry. The lowest mass tachyonic states of zero KaluzaKlein momentum $m=0$ and winding number $w=2 n+1= \pm 1$ are odd under it and therefore eliminated from the spectrum. The lowest mass scalar states in the spectrum are the antisymmetrized combinations

$$
\begin{aligned}
& \left|T_{1}>=\right| m=+1, w=1>-\mid m=-1, w=1>, \\
& \left|T_{2}>=\right| m=+1, w=-1>-\mid m=-1, w=-1>.
\end{aligned}
$$

This orientifold projection removes the closed tachyon for any radius $R$ since the mass of the states (41)

$$
M_{T}^{2}=-\frac{2}{\alpha^{\prime}}+\frac{1}{R^{2}}+\frac{R^{2}}{\alpha^{\prime 2}}=\left(\frac{1}{R}-\frac{R}{\alpha^{\prime}}\right)^{2}
$$

\footnotetext{
${ }^{3}$ A similar way of eliminating the closed string tachyon was first proposed in the context of Type O orientifolds by Sagnotti [5]. Its implementation in Scherk-Schwarz compactifications was proposed in [10].
} 
is positive and becomes zero at the self-dual value of the compact radius. It is easily checked that in Scherk-Schwarz compactifications $\Omega^{\prime}$ squares to one because $(-1)^{f_{L}+\bar{f}_{R}}=1$ in all sectors. The involution $\Omega^{\prime}$ generates $O p_{+}$-planes at $y=0$ and anti $O 8_{-}$-planes at $y=\pi R$.

These features are present in all explicit string theory examples and will become more transparent in the vacua constructed in Sections 4 and 5.

\section{String vacua in various dimensions}

In this section we present explicit string constructions realizing the Dp-brane / $\overline{O p}_{-}$-plane system introduced in Section 2. All of the explicit constructions start from a freely-acting orbifold $g$ in the type II string, containing the spacetime fermion number $(-1)^{F}$. After a radius redefinition, the orbifold $g$ becomes a periodic identification $y=y+2 \pi R$ accompanied by the spacetime fermion number operation, imposing different boundary conditions for bosons and fermions and breaking therefore supersymmetry. The orientifold operations $\Omega^{\prime}=\Omega \Pi_{y}(-1)^{f_{L}}$ and $\Omega^{\prime} g$ create O-planes of two different types. The fixed plane of $\Omega^{\prime}$ sits at the origin $y=0$ and is a standard $O_{+}$plane, whereas the fixed plane of $\Omega^{\prime} g$ sits at $y=\pi R$ and is an antiorientifold plane, due to the action of $(-1)^{F}$. More precisely, it is an $\bar{O}_{-}$plane due to the simultaneous action of $(-1)^{F}$ and $(-1)^{f_{L}}$ operations.

The simplest example of this type is nine-dimensional and was provided in [11]. A straightforward way of producing lower-dimensional examples is by taking five additional dimensions to be compact and performing T-dualities on the original nine-dimensional example. There are several reasons, however, to search for new examples. The first is that T-dualities on the $9 \mathrm{~d}$ example will produce non-chiral vacua in lower dimensions, whereas a realistic example asks for chirality. In this respect, we construct six and four-dimensional chiral examples by compactifying on orbifolds. The second reason is building models with a rich spectrum of D-branes. Our six and four-dimensional examples are based on $Z_{2}$ and $Z_{2} \times Z_{2}$ orbifold compactifications.

\subsection{String vacua in 8 dimensions}

The starting point for the eight-dimensional constructions is the $I I B$ superstring compactified on a $T^{2}$ torus, subject to the orbifold identification $g=(-1)^{F} \delta$, where $F$ is the spacetime fermion number and $\delta$ is the symmetric shift

$$
\delta: X_{i}=X_{i}+\pi R_{i}, \quad i=1,2 .
$$

We introduce for convenience the complex coordinate $z=X_{1}+i X_{2}$. Then the original circle identification $X_{i} \equiv X_{i}+2 \pi R_{i}$ and the shift operation $\delta$ are both contained in the identifications

$$
z=z+1, \quad z=z+\tau
$$


where $\tau=(1 / 2)+i\left(R_{2} / 2 R_{1}\right)$. The shift identification has thus the effect of turning the square torus into a tilted torus of complex structure $\tau$. For later use we also define the Kahler modulus $\rho=i\left(R_{1} R_{2} / 2\right)$.

The $g$ operation breaks completely supersymmetry and has no fixed points. The corresponding torus amplitude reads:

$$
\begin{aligned}
\mathcal{T} & =\left(\left|V_{8}\right|^{2}+\left|S_{8}\right|^{2}\right)\left(\Lambda_{2 m_{1}, n_{1}} \Lambda_{2 m_{2}, n_{2}}+\Lambda_{2 m_{1}+1, n_{1}} \Lambda_{2 m_{2}+1, n_{2}}\right) \\
& +\left(\left|O_{8}\right|^{2}+\left|C_{8}\right|^{2}\right)\left(\Lambda_{2 m_{1}, n_{1}+1 / 2} \Lambda_{2 m_{2}, n_{2}+1 / 2}+\Lambda_{2 m_{1}+1, n_{1}+1 / 2} \Lambda_{2 m_{2}+1, n_{2}+1 / 2}\right) \\
& -\left(V_{8} \bar{S}_{8}+S_{8} \bar{V}_{8}\right)\left(\Lambda_{2 m_{1}, n_{1}} \Lambda_{2 m_{2}+1, n_{2}}+\Lambda_{2 m_{1}+1, n_{1}} \Lambda_{2 m_{2}, n_{2}}\right) \\
& -\left(O_{8} \bar{C}_{8}+C_{8} \bar{O}_{8}\right)\left(\Lambda_{2 m_{1}, n_{1}+1 / 2} \Lambda_{2 m_{2}+1, n_{2}+1 / 2}+\Lambda_{2 m_{1}+1, n_{1}+1 / 2} \Lambda_{2 m_{2}, n_{2}+1 / 2}\right)
\end{aligned}
$$

The most natural orientifold candidate for a freely-acting orbifold is the one obtained with the operation containing one parity $\Omega^{\prime}=\Omega(-1)^{f_{L}} \Pi_{2}$. The orientifold operation introduces 08 planes, which require, for consistency, the presence of D8 branes. However, it turns out that the open string spectrum is completely supersymmetric. Indeed, the Klein amplitude

$$
\mathcal{K}=\frac{1}{2}\left(V_{8}-S_{8}\right) P_{2 m_{1}} W_{n_{2}}
$$

introduces standard $O 8_{+}$planes at $X_{2}=0, \pi R_{2}$, asking for a net number of $32 \mathrm{D} 8$ branes. The open string amplitudes

$$
\begin{aligned}
\mathcal{A} & =\frac{N^{2}}{2}\left(V_{8}-S_{8}\right) P_{m_{1}}\left(W_{n_{2}}+W_{n_{2}+1 / 2}\right) \\
\mathcal{M} & =-\frac{N}{2}\left(\hat{V}_{8}-\hat{S}_{8}\right)\left[P_{m_{1}} W_{n_{2}}+(-1)^{m_{1}} P_{m_{1}} W_{n_{2}+1 / 2}\right],
\end{aligned}
$$

are completely blind to the supersymmetry breaking present in the closed sector. The (maximal) gauge group, corresponding to putting all branes at the origin $X_{2}=0$, is $S O(16)$. These amplitudes are (after a T-duality on $X_{2}$ ) exactly the ones describing a discrete antisymmetric tensor background in the Type I superstring [6], a fact that explains the reduction of the rank of the gauge group [18. The reason behind this simple result is that geometric interpretation of O-planes / D-branes in this model asks for a T-duality in $X_{1}$. After the T-duality, the complex structure and the Kahler modulus get interchanged $\tau \leftrightarrow \rho$, such that the new ones become

$$
\tau^{\prime}=i \frac{R_{1}}{2 R_{2}^{\prime}} \quad, \quad \rho^{\prime}=\frac{1}{2}+i \frac{R_{1} R_{2}^{\prime}}{2},
$$

where $R_{2}^{\prime}$ is the T-dual radius. In particular, by using the general definition $\rho=B_{12}+i \sqrt{G}$, the presence of the quantized antisymmetric tensor $B_{12}=1 / 2$ is readily identified. This argument is equivalent to the known [19] result that an orientifold action containing a parity inversion in the presence of a nontrivial complex structure of the torus produces a reduction of the gauge 
group. It is interesting that the Klein and the open string amplitudes are exactly the same as the Type I ones, whereas the closed spectrum has soft supersymmetry, in analogy with the M-theory-type vacua constructed in the last two references of $[9$.

In order to construct a model having the pattern of O-planes put forward in Section 2, we use a different orientifold projection. In order to have a more transparent geometrical interpretation, we first rewrite the torus amplitude in a different (Scherk-Schwarz) basis, by a trivial rescaling $R_{i} \rightarrow R_{i} / 2$ of the internal radii. The torus becomes:

$$
\begin{aligned}
\mathcal{T} & =\left(\left|V_{8}\right|^{2}+\left|S_{8}\right|^{2}\right)\left(\Lambda_{m_{1}, 2 n_{1}} \Lambda_{m_{2}, 2 n_{2}}+\Lambda_{m_{1}+1 / 2,2 n_{1}} \Lambda_{m_{2}+1 / 2,2 n_{2}}\right) \\
& +\left(\left|O_{8}\right|^{2}+\left|C_{8}\right|^{2}\right)\left(\Lambda_{m_{1}, 2 n_{1}+1} \Lambda_{m_{2}, 2 n_{2}+1}+\Lambda_{m_{1}+1 / 2,2 n_{1}+1} \Lambda_{m_{2}+1 / 2,2 n_{2}+1}\right) \\
& -\left(V_{8} \bar{S}_{8}+S_{8} \bar{V}_{8}\right)\left(\Lambda_{m_{1}, 2 n_{1}} \Lambda_{m_{2}+1 / 2,2 n_{2}}+\Lambda_{m_{1}+1 / 2,2 n_{1}} \Lambda_{m_{2}, 2 n_{2}}\right) \\
& -\left(O_{8} \bar{C}_{8}+C_{8} \bar{O}_{8}\right)\left(\Lambda_{m_{1}, 2 n_{1}+1} \Lambda_{m_{2}+1 / 2,2 n_{2}+1}+\Lambda_{m_{1}+1 / 2,2 n_{1}+1} \Lambda_{m_{2}, 2 n_{2}+1}\right) .
\end{aligned}
$$

Next we consider a different orientifold projection $\Omega^{\prime}=\Omega(-1)^{f_{L}} \Pi_{1} \Pi_{2}$ containing parities in the two internal coordinates. The Klein bottle amplitude

$$
\mathcal{K}=\frac{1}{2}\left[\left(V_{8}-S_{8}\right) W_{2 n_{1}} W_{2 n_{2}}-\left(O_{8}-C_{8}\right) W_{2 n_{1}+1} W_{2 n_{2}+1}\right]
$$

introduces $O 7_{+}$-planes localized at $(0,0)$ and $\overline{O 7}_{-}$-planes in $(\pi R, \pi R)$ in the $\left(X_{1}, X_{2}\right)$ plane, which is the type of system we were searching for. The tachyonic scalar is again eliminated by the projection. The lowest possible mass scalar states have a degeneracy of four and their mass

$$
\begin{aligned}
& M_{T_{i}}^{2}=\left(\frac{1}{R_{1}}-\frac{R_{1}}{\alpha^{\prime}}\right)^{2}+\left(\frac{R_{2}}{\alpha^{\prime}}\right)^{2}, \\
& M_{S_{i}}^{2}=\left(\frac{1}{R_{2}}-\frac{R_{2}}{\alpha^{\prime}}\right)^{2}+\left(\frac{R_{1}}{\alpha^{\prime}}\right)^{2},
\end{aligned}
$$

where $i=1 \cdots 4$, is positive for any values of the compact radii.

To cancel the $\mathrm{R}-\mathrm{R}$ tadpoles we need therefore to introduce $\mathrm{N}=32$ D7-branes. The open string amplitudes for branes on top of the $O 7_{+}$planes at the origin are

$$
\begin{aligned}
\mathcal{A} & =\frac{N^{2}}{2}\left(V_{8}-S_{8}\right)\left(W_{2 n_{1}} W_{2 n_{2}}+W_{2 n_{1}+1} W_{2 n_{2}+1}\right) \\
\mathcal{M} & =-\frac{N}{2}\left[\hat{V}_{8}\left(W_{2 n_{1}} W_{2 n_{2}}+W_{2 n_{1}+1} W_{2 n_{2}+1}\right)-\hat{S}_{8}\left(W_{2 n_{1}} W_{2 n_{2}}+W_{2 n_{1}+1} W_{2 n_{2}+1}\right)\right] .
\end{aligned}
$$

For this particular configuration the gauge group is $S O(32)$, whereas putting the branes on top of the $\overline{O 7}_{-}$planes would produce an $U S p(32)$ gauge group. The second case is the one relevant for the cosmological solutions discussed in [11, 12] and in Section 5 of the present paper. The absence of the antisymmetric tensor in this case is easily explained by the fact that the geometric interpretation needs no T-dualities, and therefore the geometry is represented by the original twisted torus (7). 


\subsection{A chiral model in six dimensions}

The string vacua considered so far, if dimensionally reduced to lower dimensions, contain non-chiral fermions. Even if we are clearly interested in chiral four dimensional vacua, sixdimensional ones are the next step in this direction.

The string vacua we consider in the following contain D9 and D5 branes, and O9 and $\mathrm{O} 5$ planes which are actually $\mathrm{O} 5_{+}-\overline{\mathrm{O}}_{-}$systems. By taking three T-dualities along directions transverse to the D5 branes, we find configurations consisting of D8 branes and $08_{+}-\bar{O}_{-}$ systems, together with the BPS D6 and $06_{+}$configurations. If the D6 branes are placed democratically on top of the $O 6_{+}$planes, the resulting system has no couplings to the massless closed string modes and the effective lagrangian and the corresponding classical solution are precisely the one worked out in [11].

The construction of the model starts with a Scherk-Schwarz deformation of the SUSY model $T^{4} / \mathbb{Z}_{2}$ by $(-1)^{F} \times \delta$, with $F$ the spacetime fermion number and $\delta$ the shift $\delta X_{9}=X_{9}+\pi R_{9}$. The $\mathbb{Z}_{2}$ acts by convention in the $\left(X_{6}, X_{7}, X_{8}, X_{9}\right)$ coordinates. The torus amplitude of the model is:

$$
\begin{aligned}
\mathcal{T} & =\frac{1}{4}\left\{\left|V_{8}-S_{8}\right|^{2} \Lambda_{m, n}+\left|V_{8}+S_{8}\right|^{2}(-1)^{m} \Lambda_{m, n}\right\} \Lambda^{(3,3)}+ \\
& +\frac{1}{4}\left\{\left|O_{8}-C_{8}\right|^{2} \Lambda_{m, n+1 / 2}+\left|O_{8}+C_{8}\right|^{2}(-1)^{m} \Lambda_{m, n+1 / 2}\right\} \Lambda^{(3,3)}+ \\
& +\frac{1}{4}\left\{\left(\left|Q_{o}-Q_{v}\right|^{2}+\left|Q_{o}^{\prime}-Q_{v}^{\prime}\right|^{2}\right)\left|\frac{2 \eta}{\theta_{2}}\right|^{4}+16\left(\left|Q_{s}+Q_{c}\right|^{2}+\left|Q_{s}^{\prime}+Q_{c}^{\prime}\right|^{2}\right)\left|\frac{\eta}{\theta_{4}}\right|^{4}\right\}+ \\
& +\frac{1}{4}\left\{16\left(\left|Q_{s}-Q_{c}\right|^{2}+\left|Q_{s}^{\prime}-Q_{c}^{\prime}\right|^{2}\right)\left|\frac{\eta}{\theta_{3}}\right|^{4}\right\}
\end{aligned}
$$

where:

$$
\begin{aligned}
& Q_{o}=V_{4} O_{4}-C_{4} C_{4} \quad, \quad Q_{o}^{\prime}=V_{4} O_{4}-S_{4} S_{4}, \\
& Q_{v}=O_{4} V_{4}-S_{4} S_{4} \quad, \quad Q_{v}^{\prime}=O_{4} V_{4}-C_{4} C_{4} \text {, } \\
& Q_{s}=O_{4} C_{4}-S_{4} O_{4} \quad, \quad Q_{s}^{\prime}=O_{4} S_{4}-C_{4} O_{4} \text {, } \\
& Q_{c}=V_{4} S_{4}-C_{4} V_{4} \quad, \quad Q_{c}^{\prime}=V_{4} C_{4}-S_{4} V_{4} \text {. }
\end{aligned}
$$

The three lattice sums $\Lambda^{(3,3)}$ refer to the $\left(X_{6}, X_{7}, X_{8}\right)$ coordinates, whereas the remaining lattice sums $\left(\Lambda_{m, 2 n}\right.$, etc) refer to the coordinate $X_{9}$ involved in the supersymmetry breaking deformation. After the rescaling $R_{9} \rightarrow 2 R_{9}$ the torus amplitude becomes:

$$
\mathcal{T}=\frac{1}{2}\left\{\left(\left|V_{8}\right|^{2}+\left|S_{8}\right|^{2}\right) \Lambda_{m, 2 n}+\left(\left|O_{8}\right|^{2}+\left|C_{8}\right|^{2}\right) \Lambda_{m, 2 n+1}\right\} \Lambda^{(3,3)}-
$$




$$
\begin{aligned}
& -\frac{1}{2}\left\{\left(V_{8} \bar{S}_{8}+S_{8} \bar{V}_{8}\right) \Lambda_{m+1 / 2,2 n}+\left(O_{8} \bar{C}_{8}+C_{8} \bar{O}_{8}\right) \Lambda_{m+1 / 2,2 n+1}\right\} \Lambda^{(3,3)}+ \\
& +\frac{1}{4}\left\{\left(\left|Q_{o}-Q_{v}\right|^{2}+\left|Q_{o}^{\prime}-Q_{v}^{\prime}\right|^{2}\right)\left|\frac{2 \eta}{\theta_{2}}\right|^{4}+16\left(\left|Q_{s}+Q_{c}\right|^{2}+\left|Q_{s}^{\prime}+Q_{c}^{\prime}\right|^{2}\right)\left|\frac{\eta}{\theta_{4}}\right|^{4}\right\}+ \\
& +\frac{1}{4}\left\{16\left(\left|Q_{s}-Q_{c}\right|^{2}+\left|Q_{s}^{\prime}-Q_{c}^{\prime}\right|^{2}\right)\left|\frac{\eta}{\theta_{3}}\right|^{4}\right\}
\end{aligned}
$$

Next we construct the orientifold by gauging the discrete symmetry $\Omega^{\prime}=\Omega(-1)^{f_{L}}$, where $\Omega$ is the standard worldsheet parity operator and $(-1)^{f_{L}}$ is the worldsheet fermion number ${ }^{4}$. The corresponding Klein bottle amplitude is then:

$$
\begin{aligned}
\mathcal{K} & =\frac{1}{4}\left\{\left(V_{8}-S_{8}\right)\left(P_{m} P^{3}+W_{2 n} W^{3}\right)-\left(O_{8}-C_{8}\right) W_{2 n+1} W^{3}\right\}+ \\
& +\frac{2 \times 8}{4}\left(Q_{s}+Q_{c}-Q_{s}^{\prime}-Q_{c}^{\prime}\right)\left(\frac{\eta}{\theta_{4}}\right)^{4} .
\end{aligned}
$$

Notice that the negative parity of the untwisted sector tachyon in (19) is accompanied by a peculiar orientifold action in the twisted sector : the twisted sector is symmetrized in half of the fixed points, while it is antisymmetrized in the other half.

The untwisted closed string spectrum of the model has only massive fermions due to the Scherk-Schwarz deformation. The massless spectrum contains the bosons from the gravity multiplet, one tensor multiplet and four hyper-multiplets. The sixteen fixed points of the twisted sector contain supersymmetric multiplets. There are eight twisted hypers localized in eight fixed points and eight twisted tensor multiplets in the remaining eight fixed points.

To determine the content in O5-planes of the model we look at the transverse Klein bottle amplitude:

$$
\begin{aligned}
\widetilde{\mathcal{K}} & =\frac{2^{5}}{4}\left\{\left(v W_{e} W_{e}^{3}+\frac{1}{2 v} P P_{e}^{3}\right)\left(V_{8}-S_{8}\right)-\frac{1}{2 v}(-1)^{m} P_{m} P_{e}^{3}\left(V_{8}+S_{8}\right)\right\} \\
& +\frac{2^{5}}{4}\left\{\left(Q_{o}-Q_{v}-Q_{o}^{\prime}+Q_{v}^{\prime}\right)\left(\frac{2 \eta}{\theta_{2}}\right)^{2}\right\} .
\end{aligned}
$$

The model contains $O 9_{+}$planes, $16 O 5_{+}$and $16 \overline{O 5}_{-}$planes. The RR tadpole cancellation requires $32 D 9$ branes and $32 D 5$ branes.

Let us start with the case where all D5 branes are coincident with one $O 5_{+}$plane. The transverse annulus amplitude reads :

\footnotetext{
${ }^{4}$ The Scherk-Schwarz orientifold builded with the standard $\Omega$ projection was constructed in 9 .
} 


$$
\begin{aligned}
\widetilde{\mathcal{A}} & =\frac{2^{-5}}{4} v\left\{(N+\bar{N})^{2}\left(V_{8}-S_{8}\right) W_{2 n}-(N-\bar{N})^{2}\left(O_{8}-C_{8}\right) W_{2 n+1}\right\} W^{3}+ \\
& +\frac{2^{-5}}{4}\left\{8\left(R_{N}+R_{\bar{N}}\right)^{2}\left(Q_{s}+Q_{c}\right)\left(\frac{\eta}{\theta_{4}}\right)^{2}-8\left(R_{N}-R_{\bar{N}}\right)^{2}\left(Q_{s}^{\prime}+Q_{c}^{\prime}\right)\left(\frac{\eta}{\theta_{4}}\right)^{2}\right\}+ \\
& +\frac{2^{-5}}{4}\left\{\frac{D^{2}}{v} P^{4}\left(V_{8}-S_{8}\right)+2(N+\bar{N}) D\left(Q_{o}-Q_{v}\right)\left(\frac{2 \eta}{\theta_{2}}\right)^{2}+16 R_{D}^{2}\left(Q_{s}+Q_{c}\right)\left(\frac{\eta}{\theta_{4}}\right)^{2}\right\}+ \\
& +\frac{2^{-5}}{4}\left\{2\left(R_{N}+R_{\bar{N}}\right) R_{D}\left(Q_{s}-Q_{c}\right)\left(\frac{2 \eta}{\theta_{3}}\right)^{2}\right\}
\end{aligned}
$$

where $N+\bar{N}$ is the number of $D_{9}$ branes, $D$ is the number of the $D_{5}$ branes, while $R_{N}, R_{\bar{N}}, R_{D}$ encode the orbifold action of $\mathbb{Z}_{2}$ on the Chan Paton charges.

The transverse Möbius amplitude is obtained from factorization of $\widetilde{K}$ and $\widetilde{A}$ :

$$
\begin{aligned}
\widetilde{\mathcal{M}} & =\frac{1}{2}\left\{-(N+\bar{N}) v\left[\hat{V}_{8}(-1)^{n} W_{2 n}-\hat{S}_{8} W_{2 n}\right] W_{e}^{3}+\frac{D}{v} \hat{S}_{8} P_{e} P_{e}^{3}\right\}+ \\
& +\frac{1}{2}\left\{-D\left(\hat{Q}_{o}-\hat{Q}_{v}\right)\left(\frac{2 \hat{\eta}}{\hat{\theta}_{2}}\right)^{2}-\frac{D}{v} \hat{V}_{8} P_{2 m+1} P_{e}^{3}-(N+\bar{N})\left(\hat{S}_{4} \hat{S}_{4}-\hat{C}_{4} \hat{C}_{4}\right)\left(\frac{2 \hat{\eta}}{\hat{\theta}_{2}}\right)^{2}\right\}
\end{aligned}
$$

and the R-R tadpoles condition imply $N+\bar{N}=D=32$ and $R_{N}=R_{\bar{N}}=R_{D}=0$.

The direct amplitudes for open strings are obtained from the transverse channel by an $S$ transformation for the annulus and a $P$ transformation for the Möbius amplitude:

$$
\begin{aligned}
\mathcal{A} & =\frac{1}{8}\left\{(N+\bar{N})^{2}\left(V_{8}-S_{8}\right)\left(P_{m}+P_{m+1 / 2}\right)-(N-\bar{N})^{2}\left(V_{8}+S_{8}\right)\left(P_{m}-P_{m+1 / 2}\right)\right\} P^{3}+ \\
& +\frac{1}{8}\left\{2 D^{2}\left(V_{8}-S_{8}\right) W^{4}+4(N+\bar{N}) D\left(Q_{s}+Q_{c}\right)\left(\frac{\eta}{\theta_{4}}\right)^{2}\right\}+ \\
& +\frac{1}{8}\left\{\left(R_{N}+R_{\bar{N}}\right)^{2}\left(Q_{o}-Q_{v}\right)\left(\frac{2 \eta}{\theta_{2}}\right)^{2}-\left(R_{N}-R_{\bar{N}}\right)^{2}\left(Q_{o}^{\prime}-Q_{v}^{\prime}\right)\left(\frac{2 \eta}{\theta_{2}}\right)^{2}\right\}+ \\
& +\frac{1}{8}\left\{2 R_{D}^{2}\left(Q_{o}-Q_{v}\right)\left(\frac{2 \eta}{\theta_{2}}\right)^{2}+4\left(R_{N}+R_{\bar{N}}\right) R_{D}\left(Q_{s}-Q_{c}\right)\left(\frac{\eta}{\theta_{3}}\right)^{2}\right\} \\
\mathcal{M} & =\frac{1}{4}\left\{-(N+\bar{N})\left(\hat{V}_{8} P_{m+1 / 2}-\hat{S}_{8} P_{m}\right) P^{3}-D\left(\hat{V}_{8}(-1)^{n}-\hat{S}_{8}\right) W_{n} W^{3}\right\}+ \\
& +\frac{1}{4}\left\{(N+\bar{N})\left(\hat{S}_{4} \hat{S}_{4}-\hat{C}_{4} \hat{C}_{4}\right)\left(\frac{2 \hat{\eta}}{\hat{\theta}_{2}}\right)^{2}+D\left(\hat{Q}_{o}-\hat{Q}_{v}\right)\left(\frac{2 \hat{\eta}}{\hat{\theta}_{2}}\right)^{2}\right\}
\end{aligned}
$$

In order to have a consistent particle interpretation of the open amplitudes we have to 
parameterize $N, \bar{N}, D, R_{N}, R_{\bar{N}}, R_{D}$ in terms of the real Chan Paton multiplicites:

$$
\begin{array}{rlrl}
N & =n_{1}+n_{2} \\
R_{N} & =i\left(n_{1}-n_{2}\right), & D & =d+\bar{d}, \\
R_{D} & =i(d-\bar{d}) .
\end{array}
$$

The gauge group is $[U(8) \otimes U(8)]_{9} \otimes U(16)_{5}$. The D9 massless spectrum is nonsupersymmetric and consists of 4 scalars in the $(\mathbf{8}, \overline{\mathbf{8}}, \mathbf{1})+(\overline{\mathbf{8}}, \mathbf{8}, \mathbf{1})$. There are fermions (gaugini) in the bifundamental $(\mathbf{8}, \mathbf{8}, \mathbf{1})$ and $(\overline{\mathbf{8}}, \overline{\mathbf{8}}, \mathbf{1})$ representations. Matter fermions (of opposite chirality) are in $(\mathbf{2 8}, \mathbf{1}, \mathbf{1})+(\overline{\mathbf{2 8}}, \mathbf{1}, \mathbf{1})+(\mathbf{1}, \mathbf{2 8}, \mathbf{1})+(\mathbf{1}, \overline{\mathbf{2 8}}, \mathbf{1})$. The D5 massless spectrum is supersymmetric and has, in addition to the adjoint vector multiplet, hypermultiplets in $(\mathbf{1}, \mathbf{1}, \mathbf{1 2 0})+(\mathbf{1}, \mathbf{1}, \overline{\mathbf{1 2 0}})+(\mathbf{8}, \mathbf{1}, \overline{\mathbf{1 6}})+(\mathbf{1}, \overline{\mathbf{8}}, \overline{\mathbf{1 6}})$. The model we just constructed has all D5 branes on top of one $05_{+}$-plane. The massless spectrum on D5 branes should therefore be supersymmetric and this is indeed the case. Notice from the Chan-Paton parameterization (24) the consistency of the string amplitudes (21)-(22) in the closed channel. Indeed, it is well known from field theory arguments that in six dimensions branes cannot couple to twisted hypermultiplets. In (22) these couplings are proportional to $R_{N}+R_{\bar{N}}$ and $R_{D}$, and are indeed unphysical. On the other hand, branes can consistently couple to (the Hodge dual of) twisted tensor multiplets, and indeed in (22) the couplings of D9 branes (D5 branes in this model live in one fixed point containing one hyper), proportional to $R_{N}-R_{\bar{N}}$, are physical.

Irreducible gauge and gravitational anomalies are easily seen to cancel in this model. The reducible part of the anomaly polynomial is

$$
I_{8}=-\frac{1}{16}\left(\operatorname{tr} R^{2}-\operatorname{tr} F_{1}^{2}-\operatorname{tr} F_{2}^{2}-\operatorname{tr} F_{5}^{2}\right)^{2}-\frac{1}{4}\left(\operatorname{tr} F_{1}^{2}-\operatorname{tr} F_{2}^{2}\right)^{2}+\frac{1}{16}\left(\operatorname{tr} F_{1}^{2}+\operatorname{tr} F_{2}^{2}-\operatorname{tr} F_{5}^{2}\right)^{2},
$$

and is taken care by the generalized Green-Schwarz mechanism [20].

The geometric configurations for which the time-dependent solutions are valid correspond to moving some D5 branes on top of $O 5_{+}$planes and some on top of $\overline{O 5}_{-}$planes. Moving D5 branes from a fixed point containing closed twisted hypers to another one containing twisted tensors presents interesting subtleties in that the cylinder amplitude, even if a Wilson line deformation of the former one, leads to an amplitude that has a qualitatively different structure. For example, moving all D5 branes on top of a $\overline{O 5}_{-}$plane gives a tree-level cylinder amplitude:

$$
\begin{aligned}
\widetilde{\mathcal{A}} & =\frac{2^{-5}}{4} v\left\{(N+\bar{N})^{2}\left(V_{8}-S_{8}\right) W_{2 n}-(N-\bar{N})^{2}\left(O_{8}-C_{8}\right) W_{2 n+1}\right\} W^{3}+ \\
& +\frac{2^{-5}}{4}\left\{8\left(R_{N}+R_{\bar{N}}\right)^{2}\left(Q_{s}^{\prime}+Q_{c}^{\prime}\right)\left(\frac{\eta}{\theta_{4}}\right)^{2}-8\left(R_{N}-R_{\bar{N}}\right)^{2}\left(Q_{s}+Q_{c}\right)\left(\frac{\eta}{\theta_{4}}\right)^{2}\right\}+ \\
& +\frac{2^{-5}}{4}\left\{\frac{D^{2}}{v} P^{4}\left(V_{8}-S_{8}\right)+2(N+\bar{N}) D\left(Q_{o}-Q_{v}\right)\left(\frac{2 \eta}{\theta_{2}}\right)^{2}+16 R_{D}^{2}\left(Q_{s}^{\prime}+Q_{c}^{\prime}\right)\left(\frac{\eta}{\theta_{4}}\right)^{2}\right\}+ \\
& +\frac{2^{-5}}{4}\left\{2\left(R_{N}+R_{\bar{N}}\right) R_{D}\left(-O_{4} S_{4}-C_{4} O_{4}+V_{4} C_{4}+S_{4} V_{4}\right)\left(\frac{2 \eta}{\theta_{3}}\right)^{2}\right\}
\end{aligned}
$$


which roughly speaking exchanges twisted sector characters $Q_{s, c} \leftrightarrow Q_{s, c}^{\prime}$ and generates a completely nonsupersymmetric character in the last line. The loop open amplitudes in this case are given by:

$$
\begin{aligned}
\mathcal{A} & =\frac{1}{8}\left\{(N+\bar{N})^{2}\left(V_{8}-S_{8}\right)\left(P_{m}+P_{m+1 / 2}\right)-(N-\bar{N})^{2}\left(V_{8}+S_{8}\right)\left(P_{m}-P_{m+1 / 2}\right)\right\} P^{3}+ \\
& +\frac{1}{8}\left\{2 D^{2}\left(V_{8}-S_{8}\right) W^{4}+4(N+\bar{N}) D\left(Q_{s}+Q_{c}\right)\left(\frac{\eta}{\theta_{4}}\right)^{2}\right\}+ \\
& +\frac{1}{8}\left\{\left(R_{N}+R_{\bar{N}}\right)^{2}\left(Q_{o}^{\prime}-Q_{v}^{\prime}\right)\left(\frac{2 \eta}{\theta_{2}}\right)^{2}-\left(R_{N}-R_{\bar{N}}^{2}\right)\left(Q_{o}-Q_{v}\right)\left(\frac{2 \eta}{\theta_{2}}\right)^{2}\right\}+ \\
& +\frac{1}{8}\left\{2 R_{D}^{2}\left(Q_{o}^{\prime}-Q_{v}^{\prime}\right)\left(\frac{2 \eta}{\theta_{2}}\right)^{2}+4\left(R_{N}+R_{\bar{N}}\right) R_{D}\left(-O_{4} C_{4}+V_{4} S_{4}-S_{4} O_{4}+C_{4} V_{4}\right)\left(\frac{\eta}{\theta_{3}}\right)^{2}\right\}, \\
\mathcal{M} & =\frac{1}{4}\left\{-(N+\bar{N})\left(\hat{V}_{8} P_{m+1 / 2}-\hat{S}_{8} P_{m}\right) P^{3}+D\left(\hat{V}_{8}(-1)^{n}+\hat{S}_{8}\right) W_{n} W^{3}\right\}+ \\
& +\frac{1}{4}\left\{(N+\bar{N})\left(\hat{S}_{4} \hat{S}_{4}-\hat{C}_{4} \hat{C}_{4}\right)\left(\frac{2 \hat{\eta}}{\hat{\theta}_{2}}\right)^{2}+D\left(\hat{Q}_{o}-\hat{Q}_{v}\right)\left(\frac{2 \hat{\eta}}{\hat{\theta}_{2}}\right)^{2}\right\},
\end{aligned}
$$

and the Chan Paton parameterization and RR tadpole conditions in this case are

$$
\begin{aligned}
N & =n_{1}+n_{2}=32, & D & =d_{1}+d_{2}=32, \\
R_{N} & =n_{1}-n_{2}=0, & R_{D} & =d_{1}-d_{2}=0 .
\end{aligned}
$$

Notice again that the couplings to the twisted tensors $Q_{s, c}^{\prime}$ are physical, whereas the ones to the twisted hypers $Q_{s, c}$ are unphysical. The amplitudes (27) describe a configuration with all D5 branes on top of an $\overline{O 5}$ - plane and the gauge group becomes $[U(8) \otimes U(8)]_{9} \otimes$ $[U S p(16) \otimes U S p(16)]_{5}$. As expected, in this case both D9 and D5 massless spectra are nonsupersymmetric. The massless spectrum consists of 4 scalars in the $(\mathbf{8}, \overline{\mathbf{8}}, \mathbf{1}, \mathbf{1})+(\overline{\mathbf{8}}, \mathbf{8}, \mathbf{1}, \mathbf{1})+$ $(\mathbf{1}, \mathbf{1}, \mathbf{1 6}, \mathbf{1 6})+(\mathbf{8}, \mathbf{1}, \mathbf{1}, \mathbf{1 6})+(\mathbf{1}, \mathbf{8}, \mathbf{1 6}, \mathbf{1})$. There are fermions (gaugini) in the bifundamental $(\mathbf{8}, \mathbf{8}, \mathbf{1}, \mathbf{1})+(\overline{\mathbf{8}}, \overline{\mathbf{8}}, \mathbf{1}, \mathbf{1})+(\mathbf{1}, \mathbf{1}, \mathbf{1 6}, \mathbf{1 6})$ representations. Matter fermions (of opposite chirality) are in $(28, \mathbf{1}, \mathbf{1}, \mathbf{1})+(\overline{\mathbf{2 8}}, \mathbf{1}, \mathbf{1}, \mathbf{1})+(\mathbf{1}, \mathbf{2 8}, \mathbf{1}, \mathbf{1})+(\mathbf{1}, \overline{\mathbf{2 8}}, \mathbf{1}, \mathbf{1})+(\mathbf{1}, \mathbf{1}, \mathbf{1 2 0}, \mathbf{1})+(\mathbf{1}, \mathbf{1}, \mathbf{1}, \mathbf{1 2 0})+$ $(\mathbf{8}, \mathbf{1}, \mathbf{1 6}, \mathbf{1})+(\mathbf{1}, \mathbf{8}, \mathbf{1}, \mathbf{1 6})$. There are 2 goldstinos on D5 branes, in agreement with the considerations of [21]. By denoting $G_{1,2}$ the $U S p(16)_{i}$ D5 gauge factors, the anomaly polynomial for this string vacuum is

$$
\begin{aligned}
& I_{8}=-\frac{1}{16}\left(\operatorname{tr} R^{2}-\operatorname{tr} F_{1}^{2}-\operatorname{tr} F_{2}^{2}-\frac{1}{2} \operatorname{tr} G_{1}^{2}-\frac{1}{2} \operatorname{tr} G_{2}^{2}\right)^{2}+\frac{1}{16}\left(\operatorname{tr} F_{1}^{2}+\operatorname{tr} F_{2}^{2}-\frac{1}{2} \operatorname{tr} G_{1}^{2}-\frac{1}{2} \operatorname{tr} G_{2}^{2}\right)^{2} \\
& -\frac{1}{32}\left(\operatorname{tr} F_{1}^{2}-\operatorname{tr} F_{2}^{2}+2 \operatorname{tr} G_{1}^{2}-2 \operatorname{tr} G_{2}^{2}\right)^{2}-\frac{7}{32}\left(\operatorname{tr} F_{1}^{2}-\operatorname{tr} F_{2}^{2}\right)^{2}
\end{aligned}
$$

and is taken care again by the generalized Green-Schwarz mechanism [20].

Moving only part of the D5 branes from the origin to the twisted tensor fixed points produce a D5 branes gauge group $[U(n) \otimes U S p(16-n) \otimes U S p(16-n)]_{5}$. 


\section{Four-dimensional $Z_{2} \times Z_{2}$ vacua}

We now turn to four dimensional compactification on $T^{2} \times T^{2} \times T^{2}$ of the Type-IIB theory, orbifolded by the $Z_{2} \times Z_{2}$ action generated by the identity (we will call it " $o$ ") and the $\pi$ rotations $g:(+,-,-), f:(-,+,-), h:(-,-,+)$, where the three entries in the parentheses refer to the three internal tori, while "+" and "-" denote the two group elements of $Z_{2}$. We deform the resulting $Z_{2} \times Z_{2}$ model by $(-1)^{F} \times \delta$, with $F$ the spacetime fermion number and $\delta$ the shift $\delta X_{9}=X_{9}+\pi R_{9}$.

The torus partition function, after a standard rescaling of the $X_{9}$ coordinate in order to go to the Scherk-Schwarz basis, is given by :

$$
\begin{aligned}
\mathcal{T}= & \frac{1}{4}\left\{\Lambda _ { 1 } \Lambda _ { 2 } \Lambda _ { m , n } \left[\Lambda_{m, 2 n}\left(\left|T_{o o}^{B}\right|^{2}+\left|T_{o o}^{F}\right|^{2}\right)+\Lambda_{m, 2 n+1}\left(\left|S_{o o}^{B}\right|^{2}+\left|S_{o o}^{F}\right|^{2}\right)+\Lambda_{m+1 / 2,2 n}\left(T_{o o}^{B} \bar{T}_{o o}^{F}+\bar{T}_{o o}^{B} T_{o o}^{F}\right)\right.\right. \\
& \left.+\Lambda_{m+1 / 2,2 n+1}\left(S_{o o}^{B} \bar{S}_{o o}^{F}+\bar{S}_{o o}^{B} S_{o o}^{F}\right)\right]+\Lambda_{1}\left|\frac{4 \eta^{2}}{\theta_{2}^{2}}\right|^{2} \frac{1}{2}\left[\left|T_{o g}\right|^{2}+\left|T_{o g}^{\prime}\right|^{2}\right]+\Lambda_{2}\left|\frac{4 \eta^{2}}{\theta_{2}^{2}}\right|^{2} \frac{1}{2}\left[\left|T_{o f}\right|^{2}+\left|T_{o f}^{\prime}\right|^{2}\right] \\
& +\left|\frac{4 \eta^{2}}{\theta_{2}^{2}}\right|^{2} \Lambda_{m, n}\left[\Lambda_{m, 2 n}\left(\left|T_{o h}^{B}\right|^{2}+\left|T_{o h}^{F}\right|^{2}\right)+\Lambda_{m, 2 n+1}\left(\left|S_{o h}^{B}\right|^{2}+\left|S_{o h}^{F}\right|^{2}\right)+\Lambda_{m+1 / 2,2 n}\left(T_{o h}^{B} \bar{T}_{o h}^{F}+\bar{T}_{o h}^{B} T_{o h}^{F}\right)\right. \\
& \left.+\Lambda_{m+1 / 2,2 n+1}\left(S_{o h}^{B} \bar{S}_{o h}^{F}+\bar{S}_{o h}^{B} S_{o h}^{F}\right)\right]+\Lambda_{1}\left|\frac{4 \eta^{2}}{\theta_{4}^{2}}\right|^{2} \frac{1}{2}\left[\left|T_{g o}\right|^{2}+\left|T_{g o}^{\prime}\right|^{2}\right]+\Lambda_{2}\left|\frac{4 \eta^{2}}{\theta_{4}^{2}}\right|^{2} \frac{1}{2}\left[\left|T_{f o}\right|^{2}+\left|T_{f o}^{\prime}\right|^{2}\right] \\
& +\left|\frac{4 \eta^{2}}{\theta_{4}^{2}}\right|^{2} \Lambda_{m, n}\left[\Lambda_{m, 2 n}\left(\left|T_{h o}^{B}\right|^{2}+\left|T_{h o}^{F}\right|^{2}\right)+\Lambda_{m, 2 n+1}\left(\left|S_{h o}^{B}\right|^{2}+\left|S_{h o}^{F}\right|^{2}\right)+\Lambda_{m+1 / 2,2 n}\left(T_{h o}^{B} \bar{T}_{h o}^{F}+\bar{T}_{h o}^{B} T_{h o}^{F}\right)\right. \\
& \left.+\Lambda_{m+1 / 2,2 n+1}\left(S_{h o}^{B} \bar{S}_{h o}^{F}+\bar{S}_{h o}^{B} S_{h o}^{F}\right)\right]+\Lambda_{1}\left|\frac{4 \eta^{2}}{\theta_{3}^{2}}\right|^{2} \frac{1}{2}\left[\left|T_{g g}\right|^{2}+\left|T_{g g}^{\prime}\right|^{2}\right]+\Lambda_{2}\left|\frac{4 \eta^{2}}{\theta_{3}^{2}}\right|^{2} \frac{1}{2}\left[\left|T_{f f}\right|^{2}+\left|T_{f f}^{\prime}\right|^{2}\right] \\
& +\left|\frac{4 \eta^{2}}{\theta_{3}^{2}}\right|^{2} \Lambda_{m, n}\left[\Lambda_{m, 2 n}\left(\left|T_{h h}^{B}\right|^{2}+\left|T_{h h}^{F}\right|^{2}\right)+\Lambda_{m, 2 n+1}\left(\left|S_{h h}^{B}\right|^{2}+\left|S_{h h}^{F}\right|^{2}\right)+\Lambda_{m+1 / 2,2 n}\left(T_{h h}^{B} \bar{T}_{h h}^{F}+\bar{T}_{h h}^{B} T_{h h}^{F}\right)\right. \\
& \left.-\Lambda_{m+1 / 2,2 n+1}\left(S_{h h}^{B} \bar{S}_{h h}^{F}+\bar{S}_{h h}^{B} S_{h h}^{F}\right)\right]+\epsilon\left|\frac{8 \eta^{3}}{\theta_{2} \theta_{3} \theta_{4}}\right|^{2} \frac{1}{2}\left[\left|T_{g h}\right|^{2}+\left|T_{g h}^{\prime}\right|^{2}+\left|T_{g f}\right|^{2}+\left|T_{g f}^{\prime}\right|^{2}+\left|T_{h g}\right|^{2}\right. \\
& \left.\left.+\left|T_{h g}^{\prime}\right|^{2}+\left|T_{h f}\right|^{2}+\left|T_{h f}^{\prime}\right|^{2}+\left|T_{f g}\right|^{2}+\left|T_{f g}^{\prime}\right|^{2}+\left|T_{f h}\right|^{2}+\left|T_{f h}^{\prime}\right|^{2}\right]\right\}
\end{aligned}
$$

where we left implicit the contribution of the transverse bosons and the argument of the characters, $q=\exp (2 i \pi \tau)$, with $\tau$ the modulus of the torus and $\epsilon= \pm 1$. The choice $\epsilon=1$ defines the model without discrete torsion, while the choice $\epsilon=-1$ defines the model with discrete torsion. With the characters used in (30), the would-be tachyon is contained in $S_{o o}^{B}$ and $S_{o h}^{B}$ (see their explicit definition in the appendix). Moreover, $\Lambda_{1}\left(\Lambda_{2}\right)$ denote the lattice summations in the first (second) torus, whereas the lattice summations written separately for the two torus coordinates $\left(\Lambda_{m, n} \Lambda_{m, 2 n}\right.$,etc) refer to the third torus, the first (second) sum referring to the $X_{8}$ $\left(X_{9}\right)$ coordinate. 


\subsection{A model without discrete torsion}

We start our four-dimensional constructions by discussing the model without discrete torsion $\epsilon=1$. There are several choices actually, depending on three signs $\epsilon_{i}= \pm 1$, where $\epsilon_{i}=1$ signals typically the existence of $O 5_{+}$planes, whereas $\epsilon_{i}=-1$ that of $O 5_{-}$planes. The different possibilities are restricted by the condition

$$
\epsilon=\epsilon_{1} \epsilon_{2} \epsilon_{3} .
$$

The model relevant for the cosmological solution discussed in [12 and in the next section, constructed in this paragraph, has $\epsilon_{i}=1$. In order to consistently remove the tachyon, we use the orientifold projection $\Omega^{\prime}=\Omega(-1)^{f_{L}}$.

The direct channel Klein bottle amplitude reads:

$$
\begin{aligned}
\mathcal{K}= & \frac{1}{8}\left\{\left(P_{1} P_{2} P_{3}+\left(P_{1} W_{2}+W_{1} P_{2}\right) W_{n} W_{2 n}+W_{1} W_{2} P_{3}\right) T_{o o}-\left(P_{1} W_{2}+W_{1} P_{2}\right) W_{n} W_{2 n+1} S_{o o}\right. \\
& +16\left(\frac{\eta}{\theta_{4}}\right)^{2}\left[\left(P_{1}+W_{1}\right)\left(T_{g o}-T_{g o}^{\prime}\right)+\left(P_{2}+W_{2}\right)\left(T_{f o}-T_{f o}^{\prime}\right)\right. \\
& \left.\left.+2 P_{3} T_{h o}+2 W_{n}\left(W_{2 n} T_{h o}-W_{2 n+1} S_{h o}\right)\right]\right\}
\end{aligned}
$$

where $P_{i}\left(W_{i}\right)$ denotes the restriction of $\Lambda_{i}$ to its momentum (winding) sublattice. Notice the peculiar orientifold projection in the twisted sector, which is symmetrized in half of the orbifold fixed point and antisymmetrized in the other half, in analogy with the six-dimensional orbifold constructed in the previous section.

The untwisted massless closed string spectrum consists of the bosonic part of the $Z_{2} \times Z_{2}$ orientifold one (without discrete torsion). The twisted spectrum is $\mathcal{N}=1$ supersymmetric and consists of 32 chiral multiplets and 16 vector multiplets distributed in the 48 fixed points of the orbifold. The would-be tachyon in the spectrum is again removed by the orientifold projection, as promised, whereas the lowest mass scalars have a positive definite mass given by the formula (51).

A modular S-transformation gives the transverse channel Klein bottle amplitude:

$$
\begin{aligned}
\widetilde{\mathcal{K}}= & \frac{2^{5}}{8}\left\{\left(v_{1} v_{2} W_{1}^{e} W_{2}^{e}+\frac{1}{v_{1} v_{2}} P_{1}^{e} P_{2}^{e}\right) v_{3} W_{3}^{e} T_{o o}\right. \\
& +\left(\frac{v_{1}}{v_{2} v_{3}} W_{1}^{e} P_{2}^{e}+\frac{v_{2}}{v_{1} v_{3}} P_{1}^{e} W_{2}^{e}\right) P_{2 m}\left(P_{2 m+1} T_{o o}^{B}+P_{2 m} T_{o o}^{F}\right) \\
& +\left(\frac{2 \eta}{\theta_{2}}\right)^{2}\left[\left(v_{1} W_{1}^{e}+\frac{P_{1}^{e}}{v_{1}}\right)\left(T_{o g}-T_{o g}^{\prime}\right)+\left(v_{2} W_{2}^{e}+\frac{P_{2}^{e}}{v_{2}}\right)\left(T_{o f}-T_{o f}^{\prime}\right)+2 v_{3} W_{3}^{e} T_{o h}\right. \\
& \left.\left.+2 \frac{P_{2 m}}{v_{3}}\left(P_{2 m+1} T_{o h}^{B}+P_{2 m} T_{o h}^{F}\right)\right]\right\}
\end{aligned}
$$

\footnotetext{
${ }^{5}$ The corresponding orientifold model with the standard projection $\Omega$ and with $\epsilon_{i}=1$ was already constructed by A.Cotrone 22 .
} 
where $v_{i}$ denote the volumes of the compactified tori. The nature and the geometry of the orientifold planes is completely encoded in (33), which describes $O 9_{+}$and $16 O 5_{3,+}$ planes, parallel to the compact dimension used in the Scherk-Schwarz mechanism, together with 8 $O 5_{1,+}-\overline{O 5}_{1,-}$ and $8 O 5_{2,+}-\overline{O 5}_{2,-}$ pairs of orientifold planes, orthogonal to the same compact dimension. The RR tadpole cancellation conditions ask for a net number of 32 D9 branes, described by the Chan-Paton index $N$ in the following, as well as a net number of $32 D 5_{i}$ five-branes, of CP index $D_{i}$. To make contact with the cosmological solutions of interest for us, we perform three T-dualities, say in $X_{6}, X_{7}$ and $X_{8}$ coordinates. The D9 branes become D6 and the $D 5_{3}$ become $D 6_{3}$, both of them parallel to $X_{9}$, singled out in our construction. The $D 5_{1}$ branes become $D 8_{1}$ and the $D 5_{2}$ branes become $D 4_{2}$, both of them orthogonal to $X_{9}$. This configuration does not have the $9 \mathrm{~d}$ solution of [11] as an exact solution of the classical equations, but by smearing over the four coordinates perpendicular to the $D 5_{2}$ branes and parallel to the $D 8_{1}$ branes we get the same five dimensional lagrangian [12, (477).

If we choose the simplest configuration of D-branes at the origin of the compact space, then the tree-level (transverse) cylinder amplitude is:

$$
\begin{aligned}
\widetilde{\mathcal{A}}= & \frac{2^{-5}}{8}\left\{v_{1} v_{2} v_{3} W_{1} W_{2} W_{n}\left[(N+\bar{N})^{2} W_{2 n} T_{o o}-(N-\bar{N})^{2} W_{2 n+1} S_{o o}\right]\right. \\
& +\frac{v_{1}}{v_{2} v_{3}} W_{1} P_{2} P_{3} D_{1}^{2} T_{o o}+\frac{v_{2}}{v_{1} v_{3}} P_{1} W_{2} P_{3} D_{2}^{2} T_{o o} \\
& +\frac{v_{3}}{v_{1} v_{2}} P_{1} P_{2} W_{n}\left[\left(D_{3}+\bar{D}_{3}\right)^{2} W_{2 n} T_{o o}-\left(D_{3}-\bar{D}_{3}\right)^{2} W_{2 n+1} S_{o o}\right] \\
& +2\left(\frac{2 \eta}{\theta_{2}}\right)^{2}\left[v_{1} W_{1}(N+\bar{N}) D_{1} T_{o g}+v_{2} W_{2}(N+\bar{N}) D_{2} T_{o f}+\right. \\
& v_{3} W_{n} W_{2 n}(N+\bar{N})\left(D_{3}+\bar{D}_{3}\right) T_{o h}-v_{3} W_{n} W_{2 n+1}(N-\bar{N})\left(D_{3}-\bar{D}_{3}\right) S_{o h} \\
& \left.\left.+\frac{P_{1}}{v_{1}} D_{2}\left(D_{3}+\bar{D}_{3}\right) T_{o g}+\frac{P_{2}}{v_{2}} D_{1}\left(D_{3}+\bar{D}_{3}\right) T_{o f}+\frac{P_{3}}{v_{3}} D_{1} D_{2} T_{o h}\right]\right\} .
\end{aligned}
$$

The direct channel cylinder, obtained by an S-transformation, is

$$
\begin{aligned}
\mathcal{A}= & \frac{1}{8}\left\{\frac{P_{1} P_{2}}{2}\left[(N+\bar{N})^{2}\left(P_{m}+P_{m+1 / 2}\right) T_{o o}-(N-\bar{N})^{2}\left(P_{m}-P_{m+1 / 2}\right) T_{o o}^{B-F}\right]\right. \\
& +\left(P_{1} W_{2} W_{3} D_{1}^{2}+W_{1} P_{2} W_{3} D_{2}^{2}\right) T_{o o}+ \\
& \frac{W_{1} W_{2}}{2}\left[\left(D_{3}+\bar{D}_{3}\right)^{2}\left(P_{m}+P_{m+1 / 2}\right) T_{o o}-\left(D_{3}-\bar{D}_{3}\right)^{2}\left(P_{m}-P_{m+1 / 2}\right) T_{o o}^{B-F}\right] \\
& +2\left(\frac{\eta}{\theta_{4}}\right)^{2}\left[P_{1}(N+\bar{N}) D_{1} T_{g o}+P_{2}(N+\bar{N}) D_{2} T_{f o}\right. \\
& +\frac{1}{2}(N+\bar{N})\left(D_{3}+\bar{D}_{3}\right)\left(P_{m}+P_{m+1 / 2}\right) T_{h o}-\frac{1}{2}(N-\bar{N})\left(D_{3}-\bar{D}_{3}\right)\left(P_{m}-P_{m+1 / 2}\right) T_{h o}^{B-F} \\
& \left.\left.+W_{1} D_{2}\left(D_{3}+\bar{D}_{3}\right) T_{g o}+W_{2} D_{1}\left(D_{3}+\bar{D}_{3}\right) T_{f o}+W_{3} D_{1} D_{2} T_{h o}\right]\right\} .
\end{aligned}
$$

The tree-level channel Möbius amplitude is found, as usual, by factorization :

$$
\widetilde{\mathcal{M}}=-\frac{1}{4}\left\{\left[(N+\bar{N}) v_{1} v_{2} W_{1}^{e} W_{2}^{e}+\frac{D_{3}+\bar{D}_{3}}{v_{1} v_{2}} P_{1}^{e} P_{2}^{e}\right] v_{3}\left[(-1)^{n} \hat{T}_{o o}^{B}+\hat{T}_{o o}^{F}\right] W_{2 n}\right.
$$




$$
\begin{aligned}
& +\left(\frac{v_{1}}{v_{2} v_{3}} D_{1} W_{1}^{e} P_{2}^{e}+\frac{v_{2}}{v_{1} v_{3}} D_{2} P_{1}^{e} W_{2}^{e}\right)\left(P_{2 m} \hat{T}_{o o}^{F}+P_{2 m+1} \hat{T}_{o o}^{B}\right) \\
& +\left(\frac{2 \hat{\eta}}{\hat{\theta}_{2}}\right)^{2}\left[v_{1} W_{1}^{e}\left((N+\bar{N}) \hat{T}_{o g}^{F}+D_{1} \hat{T}_{o g}\right)+\frac{P_{1}^{e}}{v_{1}}\left(\left(D_{3}+\bar{D}_{3}\right) \hat{T}_{o g}^{F}+D_{2} \hat{T}_{o g}\right)\right. \\
& +v_{2} W_{2}^{e}\left((N+\bar{N}) \hat{T}_{o f}^{F}+D_{2} \hat{T}_{o f}\right)+\frac{P_{2}^{e}}{v_{2}}\left(\left(D_{3}+\bar{D}_{3}\right) \hat{T}_{o f}^{F}+D_{1} \hat{T}_{o f}\right) \\
& \left.\left.+v_{3} W_{3}^{e}\left(N+\bar{N}+D_{3}+\bar{D}_{3}\right)\left[-(-1)^{n} \hat{T}_{o h}^{B}+\hat{T}_{o h}^{F}\right]+\frac{1}{v_{3}}\left(D_{1}+D_{2}\right)\left(P_{2 m} \hat{T}_{o h}^{F}+P_{2 m+1} \hat{T}_{o h}^{B}\right)\right]\right\}
\end{aligned}
$$

where we have used a proper basis of "hatted characters". In order to obtain the direct Mobius amplitude we have to perform a P-transformation. The final result is

$$
\begin{aligned}
& \mathcal{M}=-\frac{1}{8}\left\{\left[(N+\bar{N}) P_{1} P_{2}+\left(D_{3}+\bar{D}_{3}\right) W_{1} W_{2}\right]\left(P_{m+1 / 2} \hat{T}_{o o}^{B}+P_{m} \hat{T}_{o o}^{F}\right)\right. \\
& +\left(D_{1} P_{1} W_{2}+D_{2} W_{1} P_{2}\right)\left[(-1)^{n} \hat{T}_{o o}^{B}+\hat{T}_{o o}^{F}\right] W_{3} \\
& -\left(\frac{2 \hat{\eta}}{\hat{\theta}_{2}}\right)^{2}\left[P_{1}\left((N+\bar{N}) \hat{T}_{o g}^{F}+D_{1} \hat{T}_{o g}\right)+W_{1}\left(\left(D_{3}+\bar{D}_{3}\right) \hat{T}_{o g}^{F}+D_{2} \hat{T}_{o g}\right)+P_{2}\left((N+\bar{N}) \hat{T}_{o f}^{F}+D_{2} \hat{T}_{o f}\right)\right. \\
& +W_{2}\left(\left(D_{3}+\bar{D}_{3}\right) \hat{T}_{o f}^{F}+D_{1} \hat{T}_{o f}\right)-\left(N+\bar{N}+D_{3}+\bar{D}_{3}\right)\left(P_{m+1 / 2} \hat{T}_{o h}^{B}-P_{m} \hat{T}_{o h}^{F}\right) \\
& \left.\left.+W_{3}\left(D_{1}+D_{2}\right)\left(\hat{T}_{o h}^{F}+(-1)^{n} \hat{T}_{o h}^{B}\right)\right]\right\} .
\end{aligned}
$$

The appropriate spacetime interpretation fixes the correct Chan-Paton parameterization to be $N=2 n, D_{i}=2 d_{i}$. The RR tadpole cancellation conditions then read:

$$
n+\bar{n}=d_{3}+\bar{d}_{3}=16 \quad, \quad d_{1}=d_{2}=16 .
$$

With the new CP factors, the one-loop open string amplitudes are given by:

$$
\begin{aligned}
\mathcal{A}= & \left(n \bar{n} P_{1} P_{2}+d_{3} \bar{d}_{3} W_{1} W_{2}\right)\left(P_{m} T_{o o}^{B}+P_{m+1 / 2} T_{o o}^{F}\right)+\frac{1}{2}\left[\left(n^{2}+\bar{n}^{2}\right) P_{1} P_{2}+\left(d_{3}^{2}+\bar{d}_{3}^{2}\right) W_{1} W_{2}\right] \times \\
& \times\left(P_{m+1 / 2} T_{o o}^{B}+P_{m} T_{o o}^{F}\right)+\left(\frac{d_{1}^{2}}{2} P_{1} W_{2} W_{3}+\frac{d_{2}^{2}}{2} W_{1} P_{2} W_{3}\right) T_{o o} \\
& +\left(\frac{\eta}{\theta_{4}}\right)^{2}\left\{\left[P_{1}(n+\bar{n}) d_{1}+W_{1} d_{2}\left(d_{3}+\bar{d}_{3}\right)\right] T_{g o}+\left[P_{2}(n+\bar{n}) d_{2}+W_{2} d_{1}\left(d_{3}+\bar{d}_{3}\right)\right] T_{f o}+\right. \\
& {\left[\left(n \bar{d}_{3}+\bar{n} d_{3}\right) P_{m}+\left(n d_{3}+\bar{n} \bar{d}_{3}\right) P_{m+1 / 2}\right] T_{h o}^{B} } \\
& \left.+\left[\left(n d_{3}+\bar{n} \bar{d}_{3}\right) P_{m}+\left(n \bar{d}_{3}+\bar{n} d_{3}\right) P_{m+1 / 2}\right] T_{h o}^{F}+2 W_{3} d_{1} d_{2} T_{h o}\right\} \\
\mathcal{M}= & -\frac{1}{4}\left\{\left[(n+\bar{n}) P_{1} P_{2}+\left(d_{3}+\bar{d}_{3}\right) W_{1} W_{2}\right]\left(P_{m+1 / 2} \hat{T}_{o o}^{B}+P_{m} \hat{T}_{o o}^{F}\right)\right. \\
& +\left(d_{1} P_{1} W_{2}+d_{2} W_{1} P_{2}\right)\left[(-1)^{n} \hat{T}_{o o}^{B}+\hat{T}_{o o}^{F}\right] W_{3} \\
& -\left(\frac{2 \hat{\eta}}{\hat{\theta}_{2}}\right)^{2}\left[P_{1}\left((n+\bar{n}) \hat{T}_{o g}^{F}+d_{1} \hat{T}_{o g}\right)+W_{1}\left(\left(d_{3}+\bar{d}_{3}\right) \hat{T}_{o g}^{F}+d_{2} \hat{T}_{o g}\right)+P_{2}\left((n+\bar{n}) \hat{T}_{o f}^{F}+d_{2} \hat{T}_{o f}\right)\right. \\
& +W_{2}\left(\left(d_{3}+\bar{d}_{3}\right) \hat{T}_{o f}^{F}+d_{1} \hat{T}_{o f}\right)-\left(n+\bar{n}+d_{3}+\bar{d}_{3}\right)\left(P_{m+1 / 2} \hat{T}_{o h}^{B}-P_{m} \hat{T}_{o h}^{F}\right) \\
& \left.\left.+W_{3}\left(d_{1}+d_{2}\right)\left(\hat{T}_{o h}^{F}+(-1)^{n} \hat{T}_{o h}^{B}\right)\right]\right\} .
\end{aligned}
$$


The gauge group is $U(8)_{9} \otimes U S p(16)_{5_{1}} \otimes U S p(16)_{5_{2}} \otimes U(8)_{5_{3}}$. Since we chose all D-branes to be at the origin of the compact space, we have coincident $D 5_{i}-O 5_{i,+}$ configurations. Since the $D 5_{1}$ and $D 5_{2}$ branes are orthogonal to the coordinate $X_{9}$ used in the Scherk-Schwarz mechanism, this must imply that their tree-level spectrum is supersymmetric. The charged matter spectrum consists in:

$$
\begin{array}{rll}
1 \text { Weyl fermion in } & : & (\mathbf{3 6}+\overline{\mathbf{3 6}}, \mathbf{1}, \mathbf{1}, \mathbf{1})+(\mathbf{1}, \mathbf{1}, \mathbf{1}, \mathbf{3 6}+\overline{\mathbf{3 6}}), \\
3 \text { Weyl fermions in } & : & (\mathbf{2 8}+\overline{\mathbf{2 8}}, \mathbf{1}, \mathbf{1}, \mathbf{1})+(\mathbf{1}, \mathbf{1}, \mathbf{1}, \mathbf{2 8}+\overline{\mathbf{2 8}}), \\
3 \text { complex scalars in } & : & (\mathbf{6 4}, \mathbf{1}, \mathbf{1}, \mathbf{1})+(\mathbf{1}, \mathbf{1}, \mathbf{1}, \mathbf{6 4}), \\
3 \text { chiral multiplets in } & : & (\mathbf{1}, \mathbf{1 2 0}, \mathbf{1}, \mathbf{1})+(\mathbf{1}, \mathbf{1}, \mathbf{1 2 0}, \mathbf{1}), \\
1 \text { Weyl fermion in } & : & (\mathbf{8}, \mathbf{1}, \mathbf{1}, \mathbf{8})+(\overline{\mathbf{8}}, \mathbf{1}, \mathbf{1}, \overline{\mathbf{8}}) \\
1 \text { complex scalar in } & : & (\mathbf{8}, \mathbf{1}, \mathbf{1}, \overline{\mathbf{8}})+(\overline{\mathbf{8}}, \mathbf{1}, \mathbf{1}, \mathbf{8}) \\
\text { chiral multiplets in } & : & (\mathbf{8}+\overline{\mathbf{8}}, \mathbf{1 6}, \mathbf{1}, \mathbf{1})+(\mathbf{1}, \mathbf{1}, \mathbf{1 6}, \mathbf{8}+\overline{\mathbf{8}})+(\mathbf{8}+\overline{\mathbf{8}}, \mathbf{1}, \mathbf{1 6}, \mathbf{1})+ \\
& & (\mathbf{1}, \mathbf{1 6}, \mathbf{1}, \mathbf{8}+\overline{\mathbf{8}})+2 \times(\mathbf{1}, \mathbf{1 6}, \mathbf{1 6}, \mathbf{1}) .
\end{array}
$$

The spectrum is non chiral and therefore the model is free of gauge and gravitational anomalies. As explained in Section 2 and as in the 6d example of the previous section, models relevant for the cosmological solutions constructed in Section 5 must contain $D 5_{i}-\overline{O 5}_{i,-}$ configurations. We need therefore to add appropriate Wilson lines to the present model. By a careful analysis of the NS-NS and RR charges of various O-planes, it can be readily realized that this is consistently done by changing the signs of the nonsupersymmetric bosonic couplings of the $D 5_{1,2}$ branes in the tree-level (transverse) open string amplitudes (34)-(36). Consequently, the massless $D 5_{1,2}$ branes spectrum become nonsupersymmetric, but the gauge group remains the same. Since the spectrum is still non chiral, we do not display it explicitly.

\subsection{A model with discrete torsion}

The case $\epsilon=-1$ in (30) define models with discrete torsion. It was known long time ago 23] that the corresponding models based on the $Z_{2} \times Z_{2}$ and without the Scherk-Schwarz deformation have no supersymmetric solution. It was later on realized 24] that the reason for it is that these models contain $O 5_{i,-}$ planes for each $\epsilon_{i}=-1$ and therefore consistency conditions ask for the introduction of $\overline{D 5}_{i}$ antibranes and supersymmetry is necessarily broken. The main advantage from a model building point of view in these models compared to the (simpler) ones without discrete torsion is that they contain $4 \mathrm{~d}$ chiral fermions. The case of most interest for us, satisfying (31) has $\left(\epsilon_{1}, \epsilon_{2}, \epsilon_{3}\right)=(-1,1,1)$. In this case, we expect configurations of $\overline{D 5}_{1}$ antibranes and $O 5_{1,-}-\overline{O 5}_{1,+}$ planes, $D 5_{2}$ branes and $O 5_{2,+}-\overline{O 5}_{2,-}$ planes and $D 5_{3}$ branes and $O 5_{3,+}$ planes, as we will explicitly check in the following. By choosing appropriate configurations of $D 5_{2}\left(D 5_{1}\right)$ branes (antibranes) on top of the corresponding orientifold plane systems, 
we encounter again the non-BPS configurations discussed in Section 2 and the corresponding cosmological solution. The case $\left(\epsilon_{1}, \epsilon_{2}, \epsilon_{3}\right)=(-1,-1,-1)$, whereas consistent as a perturbative orientifold construction, contain $\overline{D 5}_{3}$ branes and $O 5_{3,-}$ planes, which lead to more complicated classical solutions.

We skip here the (rather involved) details of the construction and consistency checks for the various amplitudes. The Klein bottle

$$
\begin{aligned}
\mathcal{K}= & \frac{1}{8}\left\{\left(P_{1} P_{2} P_{3}+\left(P_{1} W_{2}+W_{1} P_{2}\right) W_{n} W_{2 n}+W_{1} W_{2} P_{3}\right) T_{o o}-\left(P_{1} W_{2}+W_{1} P_{2}\right) W_{n} W_{2 n+1} S_{o o}\right. \\
& +16\left(\frac{\eta}{\theta_{4}}\right)^{2}\left[-\left(P_{1}-W_{1}\right)\left(T_{g o}-T_{g o}^{\prime}\right)+\left(P_{2}-W_{2}\right)\left(T_{f o}-T_{f o}^{\prime}\right)\right. \\
& \left.\left.+2 P_{3} T_{h o}-2 W_{n}\left(W_{2 n} T_{h o}-W_{2 n+1} S_{h o}\right)\right]\right\}
\end{aligned}
$$

which removes, as in all the other previous models, the closed string tachyon, contains no massless propagation for twisted fields, as is standard for models with discrete torsion.

By introducing appropriate Chan-Paton factors and a diagonal orbifold action on them, the loop channel cylinder amplitude, in the simplest case where all the branes are at the origin of the compact space, is given by

$$
\begin{aligned}
\mathcal{A}= & \frac{1}{8}\left\{\frac{P_{1} P_{2}}{2}\left[\left(N_{o}+\bar{N}_{o}\right)^{2}\left(P_{m}+P_{m+1 / 2}\right) T_{o o}-\left(N_{o}-\bar{N}_{o}\right)^{2}\left(P_{m}-P_{m+1 / 2}\right) T_{o o}^{B-F}\right]\right. \\
& +\left(P_{1} W_{2} W_{3} D_{g o}^{2}+W_{1} P_{2} W_{3} D_{f o}^{2}\right) T_{o o}+ \\
& \frac{W_{1} W_{2}}{2}\left[\left(D_{h o}+\bar{D}_{h o}\right)^{2}\left(P_{m}+P_{m+1 / 2}\right) T_{o o}-\left(D_{h o}-\bar{D}_{h o}\right)^{2}\left(P_{m}-P_{m+1 / 2}\right) T_{o o}^{B-F}\right] \\
& +2\left(\frac{\eta}{\theta_{4}}\right)^{2}\left[P_{1}\left(N_{o}+\bar{N}_{o}\right) D_{g o} T_{g o}^{\prime}+P_{2}\left(N_{o}+\bar{N}_{o}\right) D_{f o} T_{f o}\right. \\
& +\frac{N_{o}+\bar{N}_{o}}{2}\left(D_{h o}+\bar{D}_{h o}\right)\left(P_{m}+P_{m+1 / 2}\right) T_{h o}-\frac{N_{o}-\bar{N}_{o}}{2}\left(D_{h o}-\bar{D}_{h o}\right)\left(P_{m}-P_{m+1 / 2}\right) T_{h o}^{B-F} \\
& \left.+W_{1} D_{f o}\left(D_{h o}+\bar{D}_{h o}\right) T_{g o}+W_{2} D_{g o}\left(D_{h o}+\bar{D}_{h o}\right) T_{f o}^{\prime}+W_{3} D_{g o} D_{f o} S_{h o}\right] \\
& -2\left(\frac{\eta}{\theta_{3}}\right)^{2}\left[P_{1}\left(N_{g}+\bar{N}_{g}\right) D_{g g} T_{g g}^{\prime B-F}+P_{2}\left(N_{f}+\bar{N}_{f}\right) D_{f f} T_{f f}\right. \\
& +\frac{N_{h}+\bar{N}_{h}}{2}\left(D_{h h}+\bar{D}_{h h}\right)\left(P_{m}+P_{m+1 / 2}\right) T_{h h}-\frac{N_{h}-\bar{N}_{h}}{2}\left(D_{h h}-\bar{D}_{h h}\right)\left(P_{m}-P_{m+1 / 2}\right) T_{h h}^{B-F} \\
& \left.+W_{1} D_{f g}\left(D_{h g}+\bar{D}_{h g}\right) T_{g g}+W_{2} D_{g f}\left(D_{h f}+\bar{D}_{h f}\right) T_{f f}^{\prime B-F}+W_{3} D_{g h} D_{f h} S_{h h}\right] \\
& +\left(\frac{2 \eta}{\theta_{2}}\right)^{2}\left[P_{1}\left(\frac{1}{2}\left(N_{g}+\bar{N}_{g}\right)^{2}+D_{g g}^{2}\right)+W_{1}\left(\frac{1}{2}\left(D_{h g}+\bar{D}_{h g}\right)^{2}+D_{f g}^{2}\right)\right] T_{o g} \\
& -\frac{1}{2}\left(\frac{2 \eta}{\theta_{2}}\right)^{2}\left[P_{1}\left(N_{g}-\bar{N}_{g}\right)^{2}+W_{1}\left(D_{h g}-\bar{D}_{h g}\right)^{2}\right] T_{o g}^{\prime} \\
& +\left(\frac{2 \eta}{\theta_{2}}\right)^{2}\left[P_{2}\left(\frac{1}{2}\left(N_{f}+\bar{N}_{f}\right)^{2}+D_{f f}^{2}\right)+W_{2}\left(\frac{1}{2}\left(D_{h f}+\bar{D}_{h f}\right)^{2}+D_{g f}^{2}\right)\right] T_{o f}
\end{aligned}
$$




$$
\begin{aligned}
& -\frac{1}{2}\left(\frac{2 \eta}{\theta_{2}}\right)^{2}\left[P_{2}\left(N_{f}-\bar{N}_{f}\right)^{2}+W_{2}\left(D_{h f}-\bar{D}_{h f}\right)^{2}\right] T_{o f}^{\prime} \\
& +\left(\frac{2 \eta}{\theta_{2}}\right)^{2}\left[\frac{1}{2}\left(P_{m}+P_{m+1 / 2}\right)\left(\left(N_{h}+\bar{N}_{h}\right)^{2}+\left(D_{h h}+\bar{D}_{h h}\right)^{2}\right)+W_{3}\left(D_{g h}^{2}+D_{f h}^{2}\right)\right] T_{o h} \\
& -\left(\frac{2 \eta}{\theta_{2}}\right)^{2} \frac{1}{2}\left(P_{m}-P_{m+1 / 2}\right)\left[\left(\left(N_{h}-\bar{N}_{h}\right)^{2}+\left(D_{h h}-\bar{D}_{h h}\right)^{2}\right)\right] T_{o h}^{B-F} \\
& +\frac{4 i \eta^{3}}{\theta_{2} \theta_{3} \theta_{4}}\left[\left(N_{g}+\bar{N}_{g}\right)\left(-D_{f g} T_{f g}+\frac{D_{h g}+\bar{D}_{h g}}{2} T_{h g}\right)-\frac{1}{2}\left(N_{g}-\bar{N}_{g}\right)\left(D_{h g}-\bar{D}_{h g}\right) T_{h g}^{B-F}\right. \\
& -\left(N_{f}+\bar{N}_{f}\right)\left(D_{g f} T_{g f}^{\prime B-F}+\frac{D_{h f}+\bar{D}_{h f}}{2} T_{h f}\right)+\frac{1}{2}\left(N_{f}-\bar{N}_{f}\right)\left(D_{h f}-\bar{D}_{h f}\right) T_{h f}^{B-F} \\
& +\left(N_{h}+\bar{N}_{h}\right)\left(D_{g h} T_{g h}^{\prime}-D_{f h} T_{f h}\right) \\
& -D_{g g}\left(D_{h g}+\overline{D_{h g}}\right) T_{f g}^{\prime B-F}+D_{g g} D_{f g} S_{h g}-D_{f f} D_{g f} S_{h f} \\
& \left.\left.-D_{f f}\left(D_{h f}+\overline{D_{h f}}\right) T_{g f}+\left(D_{h h}+\overline{D_{h h}}\right)\left(D_{f h} T_{g h}-D_{g h} T_{f h}^{\prime}\right)\right]\right\}
\end{aligned}
$$

The loop channel Mobius amplitude is

$$
\begin{aligned}
& \mathcal{M}=-\frac{1}{8}\left\{\left[\left(N_{o}+\bar{N}_{o}\right) P_{1} P_{2}+\left(D_{h o}+\bar{D}_{h o}\right) W_{1} W_{2}\right]\left(P_{m+1 / 2} \hat{T}_{o o}^{B}+P_{m} \hat{T}_{o o}^{F}\right)\right. \\
& -D_{g o} P_{1} W_{2}\left[(-1)^{n} \hat{T}_{o o}^{B}-\hat{T}_{o o}^{F}\right] W_{3}+D_{f o} W_{1} P_{2}\left[(-1)^{n} \hat{T}_{o o}^{B}+\hat{T}_{o o}^{F}\right] W_{3} \\
& -\left(\frac{2 \hat{\eta}}{\hat{\theta}_{2}}\right)^{2}\left[P_{1}\left(-\left(N_{o}+\bar{N}_{o}\right) \hat{T}_{o g}^{F}+D_{g o} \hat{T}_{o g}^{\prime}\right)+W_{1}\left(\left(D_{h o}+\bar{D}_{h o}\right) \hat{T}_{o g}^{F}+D_{f o} \hat{T}_{o g}\right)+\right. \\
& P_{2}\left(\left(N_{o}+\bar{N}_{o}\right) \hat{T}_{o f}^{F}+D_{f o} \hat{T}_{o f}\right) \\
& +W_{2}\left(-\left(D_{h o}+\bar{D}_{h o}\right) \hat{T}_{o f}^{F}+D_{g o} \hat{T}_{o f}^{\prime}\right)-\left(N_{o}+\bar{N}_{o}+D_{h o}+\overline{D_{h o}}\right)\left(P_{m+1 / 2} \hat{T}_{o h}^{B}-P_{m} \hat{T}_{o h}^{F}\right) \\
& \left.\left.+W_{3} D_{g o}\left(-\hat{T}_{o h}^{F}+(-1)^{n} \hat{T}_{o h}^{B}\right)-W_{3} D_{f o}\left(\hat{T}_{o h}^{F}+(-1)^{n} \hat{T}_{o h}^{B}\right)\right]\right\} .
\end{aligned}
$$

The appropriate spacetime interpretation fixes the correct Chan-Paton parameterization to be

$$
\begin{array}{lll}
N_{o}=n_{1}+n_{2}+n_{3}+n_{4} & , \quad & N_{g}=n_{1}+n_{2}-n_{3}-n_{4}, \\
N_{f}=i\left(n_{1}-n_{2}+n_{3}-n_{4}\right) & , & N_{h}=i\left(n_{1}-n_{2}-n_{3}+n_{4}\right), \\
D_{g o}=a+b+c+d & , & D_{g g}=a+b-c-d, \\
D_{g f}=a-b+c-d & , & D_{g h}=a-b-c+d, \\
D_{f o}=o+g+\bar{o}+\bar{g} & , & D_{f g}=i(o-g-\bar{o}+\bar{g}), \\
D_{f f}=i(o+g-\bar{o}-\bar{g}) & , & D_{f h}=o-g+\bar{o}-\bar{g}, \\
D_{h o}=d_{1}+d_{2}+d_{3}+d_{4} & , & D_{h g}=i\left(d_{1}+d_{2}-d_{3}-d_{4}\right), \\
D_{h f}=d_{1}-d_{2}+d_{3}-d_{4} & , & D_{h h}=-i\left(d_{1}-d_{2}-d_{3}+d_{4}\right) .
\end{array}
$$

The RR tadpole cancellation conditions then read:

$$
\begin{aligned}
& N_{o}+\bar{N}_{o}=D_{g o}=D_{f o}=D_{h o}+\overline{D_{h o}}=32, \\
& N_{g}=\cdots D_{h h}=0 .
\end{aligned}
$$


The spectrum of the model is chiral, with a gauge group $\left[U(4)^{4}\right]_{9} \otimes[U S p(8)]_{5_{1}}^{4} \otimes\left[U(8)^{2}\right]_{5_{2}} \otimes$ $\left[U(4)^{4}\right]_{5_{3}}$. We worked out in detail the spectrum, but in order to save space, we display here only the chiral fermionic spectrum of this model. The chiral part of the spectrum arises in the $D 9-D 5_{i}$ and the $D 5_{i}-D 5_{j}$ intersections in the following representations of the appropriate bifundamental gauge group

$$
\begin{aligned}
& 9-5_{1}:(\mathbf{4}, \mathbf{1}, \mathbf{1}, \mathbf{1} ; \mathbf{8}, \mathbf{1}, \mathbf{1}, \mathbf{1})+(\overline{\mathbf{4}}, \mathbf{1}, \mathbf{1}, \mathbf{1} ; \mathbf{1}, \mathbf{8}, \mathbf{1}, \mathbf{1})+(\mathbf{1}, \mathbf{4}, \mathbf{1}, \mathbf{1} ; \mathbf{1}, \mathbf{8}, \mathbf{1}, \mathbf{1})+(\mathbf{1}, \overline{\mathbf{4}}, \mathbf{1}, \mathbf{1} ; \mathbf{8}, \mathbf{1}, \mathbf{1}, \mathbf{1}) \\
& +(1,1,4,1 ; 1,1,8,1)+(1,1, \overline{4}, 1 ; 1,1,1,8)+(1,1,1,4 ; 1,1,1,8)+(1,1,1, \overline{4} ; 1,1,8,1) \\
& 9-5_{2}:(\mathbf{4}, \mathbf{1}, \mathbf{1}, \mathbf{1} ; \overline{\mathbf{8}}, \mathbf{1})+(\overline{\mathbf{4}}, \mathbf{1}, \mathbf{1}, \mathbf{1} ; \mathbf{1}, \mathbf{8})+(\mathbf{1}, \mathbf{4}, \mathbf{1}, \mathbf{1} ; \mathbf{1}, \mathbf{8})+(\mathbf{1}, \overline{4}, \mathbf{1}, \mathbf{1} ; \overline{\mathbf{8}}, \mathbf{1}) \\
& +(1,1,4,1 ; 1, \overline{8})+(1,1, \overline{4}, 1 ; 8,1)+(1,1,1,4 ; 8,1)+(1,1,1, \overline{4} ; 1, \overline{8}) \\
& 9-5_{3}:(\mathbf{4}, \mathbf{1}, \mathbf{1}, \mathbf{1} ; \mathbf{4}, \mathbf{1}, \mathbf{1}, \mathbf{1})+(\overline{\mathbf{4}}, \mathbf{1}, \mathbf{1}, \mathbf{1} ; \mathbf{1}, \mathbf{1}, \mathbf{1}, \overline{4})+(\mathbf{1}, \mathbf{4}, \mathbf{1}, \mathbf{1} ; \mathbf{1}, \mathbf{4}, \mathbf{1}, \mathbf{1})+(\mathbf{1}, \overline{4}, \mathbf{1}, \mathbf{1} ; \mathbf{1}, \mathbf{1}, \overline{4}, \mathbf{1}) \\
& +(1,1,4,1 ; 1,1,4,1)+(1,1, \overline{4}, 1 ; 1, \overline{4}, 1,1)+(1,1,1,4 ; 1,1,1,4)+(1,1,1, \overline{4} ; \overline{4}, 1,1,1) \\
& 5_{1}-5_{2}:(\mathbf{8}, \mathbf{1}, \mathbf{1}, \mathbf{1} ; \overline{\mathbf{8}}, \mathbf{1})+(\mathbf{1}, \mathbf{8}, \mathbf{1}, \mathbf{1} ; \mathbf{1}, \mathbf{8})+(\mathbf{1}, \mathbf{1}, \mathbf{8}, \mathbf{1} ; \mathbf{1}, \overline{\mathbf{8}})+(\mathbf{1}, \mathbf{1}, \mathbf{1}, \mathbf{8} ; \mathbf{8}, \mathbf{1})+ \\
& 5_{1}-5_{3}:(8,1,1,1 ; 4,1,1,1)+(8,1,1,1 ; 1,1, \overline{4}, 1)+(1,8,1,1 ; 1,4,1,1)+(1,8,1,1 ; 1,1,1, \overline{4}) \\
& +(1,1,8,1 ; 1,1,4,1)+(1,1,8,1 ; \overline{4}, 1,1,1)+(1,1,1,8 ; 1,1,1,4)+(1,1,1,8 ; 1, \overline{4}, 1,1) \\
& 5_{2}-5_{3}:(\mathbf{8}, \mathbf{1} ; \mathbf{1}, \mathbf{1}, \mathbf{1}, \mathbf{4})+(\mathbf{8}, \mathbf{1} ; \mathbf{1}, \overline{\mathbf{4}}, \mathbf{1}, \mathbf{1})+(\overline{\mathbf{8}}, \mathbf{1} ; \mathbf{4}, \mathbf{1}, \mathbf{1}, \mathbf{1})+(\overline{\mathbf{8}}, \mathbf{1} ; \mathbf{1}, \mathbf{1}, \overline{\mathbf{4}}, \mathbf{1}) \\
& +(\mathbf{1}, \mathbf{8} ; \mathbf{1}, \mathbf{4}, \mathbf{1}, \mathbf{1})+(\mathbf{1}, \mathbf{8} ; \mathbf{1}, \mathbf{1}, \mathbf{1}, \overline{4})+(\mathbf{1}, \overline{8} ; \mathbf{1}, \mathbf{1}, 4, \mathbf{1})+(\mathbf{1}, \overline{\mathbf{8}} ; \overline{4}, \mathbf{1}, \mathbf{1}, \mathbf{1}) \text {. }
\end{aligned}
$$

Irreducible gauge anomalies are easily seen to cancel, whereas mixed anomalies are taken care by the four-dimensional Green-Schwarz mechanism involving closed sector twisted fields. The model described so far has all branes at the origin of the compact space and therefore coincident $\overline{D 5}_{1}-O 5_{1,-}$ and $D 5_{2}-O 5_{2,+}$ systems. In order to make contact with our cosmological solutions, we can add Wilson lines on the $\overline{D 5}_{1}$ and the $D 5_{2}$ branes, in order to get coincident

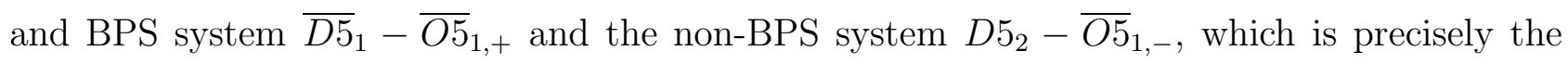
system we are searching for.

\section{Four dimensional cosmological solutions, nonpertur- bative dynamics and moduli stabilization}

In [12] we compactified and T-dualized the 9d time-dependent solution found in [11] and we found cosmological solutions for D3 branes, moving with a constant velocity in a 5d static bulk spacetime, the bulk being supersymmetric and therefore the metric free of singularities in the transverse coordinate. The five dimensional lagrangian that describes the T-dual solution with D3-branes ${ }^{6}$ is:

$$
S_{5}=\frac{1}{2 \kappa_{5}^{2}} \int d^{5} x \sqrt{-g}\left[R^{(5)}-\frac{1}{2}(\partial \Phi)^{2}-\frac{40}{3}(\partial \sigma)^{2}-\frac{1}{2 \times 5 !} e^{\frac{40 \sigma}{3}} F_{5}^{2}\right]
$$

\footnotetext{
${ }^{6}$ We remove prime indices on the T-dual fields, compared with [12].
} 


$$
\begin{aligned}
& -\int_{X_{5}=0} d^{4} x\left[\sqrt{-\gamma} T_{0} e^{-\frac{20 \sigma}{3}}+q_{0} A_{4}+\cdots\right] \\
& -\int_{X_{5}=v_{1} X_{0}} d^{4} x\left[\sqrt{-\gamma}\left(T_{1} e^{-\frac{20 \sigma}{3}}+\frac{r_{c}^{5}}{g^{2}} e^{-\Phi} \operatorname{tr} F^{2}\right)+q_{1} A_{4}+\cdots\right] .
\end{aligned}
$$

The classical solution of (47) is [12]

$$
\begin{aligned}
& d s_{5}^{2}=\left[G_{0}+\frac{3\left|q_{0}\right| \kappa^{2}}{2}\left|X_{5}\right|\right]^{\frac{2}{9}}\left[\delta_{\mu \nu} d x^{\mu} d x^{\nu}-d X_{0}^{2}+d X_{5}^{2}\right] \\
& e^{\sigma}=r_{c}\left[G_{0}+\frac{3\left|q_{0}\right| \kappa^{2}}{2}\left|X_{5}\right|\right]^{\frac{1}{6}} \quad, \quad e^{\Phi}=\text { const. }
\end{aligned}
$$

with $r_{c}$ being a constant radius parameter, the coordinates $\left(X_{0}, X_{5}\right)$ being subject to a boost identification $\left(X_{0} \pm X_{5}\right)=\exp (2 \xi)\left(X_{0} \pm X_{5}\right)$, with the boost parameter $\xi$ determined by the mismatch between the tension and the charge of the non-BPS system [11, 12] $q_{1}$ ch $\xi=T_{1}$ as defined in section 2 and the velocity of the non-BPS system being $v_{1}=t h \xi$.

By taking appropriate T-dualities, the lower dimensional orbifold examples we constructed in the Sections 3 and 4 share the same cosmological solution (48), with the advantage of allowing for nontrivial dynamics and chiral fermions on the boundary branes. This is due mostly to the fact that the twisted closed fields in the $6 \mathrm{~d}$ and $4 \mathrm{~d}$ examples of Sections 3,4 have a zero net coupling to the $\mathrm{D}$ branes $^{7}$ and therefore can be consistently set to zero in what follows. The compactified cosmological solution of [12] still had flat directions and this is most probably phenomenologically unacceptable. Fortunately, there is a simple argument showing that the classical solution (48) is valid for a large but finite time evolution. First of all, it is readily seen from (48) that the tree-level gauge couplings on the non-BPS system, moving with a constant velocity in the static bulk background, are independent of time. This result is corrected by a one-loop Weyl anomaly. Indeed, one of the steps undertaken in [12] was a Weyl rescaling in 5d from the string frame to the Einstein frame:

$$
g_{\alpha \beta}^{(5)}=\exp \left(\frac{\Phi}{2}-\frac{10 \sigma}{3}\right) g_{E, \alpha \beta}^{(5)},
$$

where we remind the reader that we removed in the present paper the primes on the T-dual fields compared to [12. This Weyl transformation is seen by the D3 brane fields as a 4d Weyl transformation, which is subject to the standard one-loop anomaly. The Weyl anomaly corrected gauge couplings become

$$
\frac{1}{g_{Y M}^{2}}=\frac{r_{c}^{5}}{g^{2}} e^{-\Phi}+\left(\frac{20 \sigma}{3}-\Phi\right) \frac{b_{1}}{32 \pi^{2}} .
$$

Since, by using (48) on the non-BPS system trajectory $X_{5}=v_{1} X_{0}$, the breathing mode $\sigma$ is increasing in time, this reflects in an effective logarithmic (Einstein proper) time dependence

\footnotetext{
${ }^{7}$ The twisted sector tensors in the $6 \mathrm{~d}$ model have actually a physical coupling, with opposite signs to the two D9 and/or D5 factors, which cancel each other and produce no net source in the field eqs.
} 
of the gauge couplings

$$
\frac{1}{g_{Y M}^{2}\left(\tau_{E}\right)}=\frac{1}{g_{Y M, 0}^{2}}+\frac{b_{1}}{32 \pi^{2}} \ln \left(\frac{\tau_{E}}{\tau_{E, 0}}\right) .
$$

It is interesting to notice the analogy of (51) with the one-loop renormalization group equations, but with energy replace by time. In the toroidal compactified model and a large class of other examples, the non-BPS system has a matter content corresponding to an asymptotically-free gauge group $b_{1}<0$. This means that, after a (exponentially) long time evolution, the nonBPS system enters a nonperturbative regime which must stop the time evolution or, in any case, must invalid the simple solution (48) combined with the constant velocity trajectory $X_{5}=v_{1} X_{0}$. The logarithmic nature of the time evolution is crucial for the cosmological realization of the hierarchies discussed in [12]. Indeed, if the time dependence was a powerlaw, the gauge coupling was either going very fast to zero (for $b_{1}>0$ ), either the system was entering, within a time evolution of the order of the Planck time, a nonperturbative regime, before generating any interesting physical effect. We believe (but we have no proof of this statement) that the tree-level bulk supersymmetry plays a crucial role in generating the desired logarithmic time dependence. The time $\tau_{\max }$ where this happens and the corresponding maximal value of the radii of the internal five-torus are

$$
\tau_{\max }=\tau_{E, 0} \exp \left\{\frac{32 \pi^{2}}{\left|b_{1}\right| g_{Y M, 0}^{2}}\right\} \quad, \quad e^{\sigma_{\max }}=R_{c} \exp \left\{\frac{24 \pi^{2}}{5\left|b_{1}\right| g_{Y M, 0}^{2}}\right\},
$$

with $R_{c}$ the initial value of the compact radii. As seen in (52), the internal radius becomes exponentially large before entering the nonperturbative regime on the non-BPS system, supporting the perturbative nature of the hierarchies proposed in [12. Any mechanism of moduli stabilization, in order to be viable, must produce a value of the compact radius smaller than its maximally admitted one (52). Going one step further, by adding the standard one-loop RGE contribution to find the full one-loop (energy-dependent) gauge couplings, we find

$$
\frac{1}{g_{Y M}^{2}(\mu)}=\frac{r_{c}^{5}}{g^{2}} e^{-\Phi}+\frac{b_{1}}{8 \pi^{2}} \ln \left(\frac{\Lambda_{0} \exp (-\Phi / 4+5 \sigma / 3)}{\mu}\right),
$$

where $\Lambda_{0}$ is the (string scale) UV cutoff. Notice that the effect of the time dependence can be absorbed into a time growth of the UV cutoff. The scalar potential induced by the gaugino condensation can be estimated, as usual, as the fourth power of the energy scale where couplings become strong

$$
V \sim \Lambda^{4}=\Lambda_{0}^{4} \exp \left(\frac{20 \sigma}{3}-\Phi\right) \exp \left\{-\frac{32 \pi^{2}}{\left|b_{1}\right| g_{Y M, 0}^{2}}\right\} \equiv \alpha \exp \left(\frac{20 \sigma}{3}\right)
$$

where

$$
\alpha \equiv \Lambda_{0}^{4} \exp \left(-\Phi_{0}\right) \exp \left\{-\frac{32 \pi^{2}}{\left|b_{1}\right| g_{Y M, 0}^{2}}\right\}
$$


In the last step, we anticipated that a constant dilaton is a solution of the brane dynamics (for example, as we will discuss in more details later on, gaugino condensation combined with additional NS-NS fluxes [25]), whereas by an appropriate combination of NS-NS and RR fluxes 14 it can be stabilized. An heuristic argument suggests that the nonperturbative potential (54) can stabilize the radius field $\sigma$. Indeed, considering the already existing potential (coming from the tension) and adding it to (54), we find

$$
V_{\text {tot }}=T_{1} \exp \left(-\frac{20 \sigma}{3}\right)+\alpha_{1} \exp \left(\frac{20 \sigma}{3}\right)
$$

which has a minimum of the order $\exp \left(40 \sigma_{0} / 3\right) \sim\left(T_{1} / \alpha_{1}\right)>>1$. By using (55), we learn that this is an exponentially large value, as required for generating hierarchies advocated in 12. It is therefore very important that $\alpha$ is much smaller than its naive value $\alpha<<\Lambda_{0}^{4}$. Moreover, $\sigma_{0}<<\sigma_{\max }$ and therefore the stabilization procedure is under control. The more detailed study in the following shows that the correct value of $\sigma_{0}$, obtained by solving the field equations, has indeed the order of magnitude of the minimum coming from (56), but we actually need additional terms in the scalar potential combining with the gaugino condensation into a perfect square. The resulting numerical coefficient will be slightly different as well.

In order to stabilize moduli we need to look more closely on the possible nonperturbative dynamics. There are two possibilities that we will consider in the following :

(i) Brane potentials.

Nonperturbative effects like gaugino condensation [25] on D-branes or loop perturbative effects generated by supersymmetry breaking on some D-branes naturally generate potentials for the closed (bulk) fields localized on the D-branes. They were already invoked some time ago in a phenomenological approach by Goldberger and Wise [13 in connection with moduli stabilization. In our case, we argued that these nonperturbative effects are naturally triggered by the time evolution. The induced potentials, called $V_{i}$ in what follows, where $i=0,1$ index the two spacetime boundaries, change our cosmological solutions in a way that is the main concern of this section.

(ii) NS-NS and RR fluxes.

Our perturbative orientifold models containing (after T-dualities) D3/O3 and D7/O7 branes and O-planes allow for the introduction of NS-NS and RR fluxes, along the lines of [14. Indeed, while the closed string spectrum and the orientifold projection in the vacua we considered are different compared to the simple IIB orientifold considered in [14, the massless untwisted spectrum contains the same RR and NS-NS fields. The additional left world-sheet fermion number in our orientifold projection has a trivial action on them and the analysis of possible fluxes to add is similar to [14 and, in context of supersymmetric orbifold cousins of the ones we discuss, was performed in [26]. Fluxes have the effect of generating potentials (which are however not brane-localized) for closed string fields [27] and to stabilize the dilaton and the 
complex structure moduli.

In order to stabilize moduli fields we add therefore potentials on the boundaries generated by nonperturbative and/or perturbative effects :

$$
S_{V}=-\int_{y=0} d^{4} x \sqrt{-\gamma} V_{0}(\sigma, \Phi)-\int_{y=y_{1}} d^{4} x \sqrt{-\gamma} V_{1}(\sigma, \Phi)
$$

where in what follows we will mostly consider (55), (56). As we will see, in the cases we are discussing, (57) does not stabilize the dilaton, which is still a flat direction of the full effective action. whereas suitable fluxes can stabilize it.

Appropriate brane potentials will stabilize the $\sigma$ and $y_{1}$ moduli fields. The non-BPS brane, which was moving into a noncompact space subject to a boost identification [11, 12, will stop moving. Stabilization of the distance between the two boundary branes means that the coordinate $X_{5}$ becomes compact again and will be denoted by $y$ in the following. The stabilization consists in our case in finding explicit solutions to the field eqs. for the lagrangian (47) + (57) and imposing appropriate boundary conditions at the position of the boundary branes $y=0$ and $y=y_{1}$.

We are in what follows searching for solutions of the field equations of the form:

$$
d s_{5}^{2}=e^{2 A(y)} g_{\mu \nu} d x^{\mu} d x^{\nu}+e^{2 B(y)} d y^{2},
$$

with $g_{\mu \nu}$ being the Minkowski metric, $\eta_{\mu \nu}$, or the de Sitter metric, $\operatorname{diag}\left(-1, e^{2 \sqrt{\Lambda} t} \delta_{i j}\right)$.

\subsection{Minkowski solution}

We are using the ansatz :

$$
\begin{aligned}
d s_{5}^{2} & =e^{2 A(y)} \eta_{\mu \nu} d x^{\mu} d x^{\nu}+e^{2 B(y)} d y^{2}, \\
F_{5} & =\tilde{f}(y) \epsilon_{5} \quad, \quad \sigma=\sigma(y), \quad \Phi=\Phi_{0}=\text { const. }
\end{aligned}
$$

where $\epsilon_{5}$ is the five-dimensional volume form. The equation of motion of the five form has the solution:

$$
\tilde{f}=-q_{0} k_{5}^{2} e^{4 A+B-\frac{40}{3} \sigma} \epsilon(y),
$$

where $\epsilon(y)$ is an odd $2 y_{1}$-periodic function and $\epsilon(y)=1$ when $y$ is between 0 and $y_{1}$.

Replacing this solution in the Einstein, $\sigma$ and $\Phi$ equations, we obtain ${ }^{8}$ :

\footnotetext{
${ }^{8}$ The notation we use is ${ }^{\prime}=\frac{d}{d y}$.
} 


$$
\begin{aligned}
& 3 A^{\prime \prime}+6 A^{\prime 2}-3 A^{\prime} B^{\prime}+\frac{20}{3} \sigma^{\prime 2}+\frac{1}{4}\left(k_{5}^{2} q_{0}\right)^{2} e^{2 B-\frac{40}{3} \sigma}=-k_{5}^{2} e^{B-\frac{20}{3} \sigma}\left[T_{0} \delta(y)+T_{1} \delta\left(y-y_{1}\right)\right]- \\
& -k_{5}^{2} e^{B}\left[V_{0} \delta(y)+V_{1} \delta\left(y-y_{1}\right)\right] \\
& 6 A^{\prime 2}-\frac{20}{3} \sigma^{\prime 2}+\frac{1}{4}\left(k_{5}^{2} q_{0}\right)^{2} e^{2 B-\frac{40}{3} \sigma}=0 \\
& \sigma^{\prime \prime}+4 A^{\prime} \sigma^{\prime}-B^{\prime} \sigma^{\prime}+\frac{1}{4}\left(k_{5}^{2} q_{0}\right)^{2} e^{2 B-\frac{40}{3} \sigma}=-\frac{k_{5}^{2}}{2} e^{B-\frac{20}{3} \sigma}\left[T_{0} \delta(y)+T_{1} \delta\left(y-y_{1}\right)\right]+ \\
& +\frac{3}{40} k_{5}^{2} e^{B}\left[\delta(y) \frac{\partial V_{0}}{\partial \sigma}+\delta\left(y-y_{1}\right) \frac{\partial V_{1}}{\partial \sigma}\right] \\
& \Phi^{\prime \prime}+4 A^{\prime} \Phi^{\prime}=2 k_{5}^{2} e^{B}\left[\delta(y) \frac{\partial V_{0}}{\partial \Phi}+\delta\left(y-y_{1}\right) \frac{\partial V_{1}}{\partial \Phi}\right] .
\end{aligned}
$$

By a change of coordinate $y$ we can fix the gauge to $B=20 \sigma / 3$. The equation (61) shows that $A^{\prime}$ and $\sigma^{\prime}$ can be parametrized by a function $f$ as follows :

$$
A^{\prime}= \pm \frac{z}{\sqrt{6}} \operatorname{shf} \epsilon(y) \quad, \quad \sigma^{\prime}= \pm z \sqrt{\frac{3}{20}} \operatorname{chf} \epsilon(y)
$$

where $z=\frac{1}{2} k_{5}^{2}\left|q_{0}\right|$.

The $(+,-)$ and $(-,-)$ solutions are incompatible with the boundary conditions below and will not be discussed anymore. The bulk part of the two remaining equations in (61) is the same, as it should, and takes the form:

$$
\sqrt{3} f^{\prime} \pm 2 \sqrt{2} z \operatorname{ch} f-2 \sqrt{5} z \operatorname{sh} f=0
$$

where the $+\operatorname{sign}$ in (63) corresponds to the $(+,+)$ case whereas the - sign corresponds to the case $(-,+)$ in (62).

The solutions of (63) with + sign are :

$$
e^{f}=-a \operatorname{th}(z|y|+C) \quad, \quad \text { and } \quad e^{f}=-a \operatorname{cth}(z|y|+C)
$$

whereas the ones with - sign are

$$
\begin{aligned}
& e^{f}=-a^{-1} \operatorname{th}(z|y|+C) \quad, \quad \text { and } e^{f}=-a^{-1} \operatorname{cth}(z|y|+C), \\
& \text { where } \quad a=\frac{\sqrt{5}+\sqrt{2}}{\sqrt{3}}
\end{aligned}
$$

and $C$ is an integration constant.

Next we have to impose the boundary conditions given by the boundary D-branes/O-planes

$$
\sqrt{\frac{3}{2}}\left|q_{0}\right| \operatorname{sh} f=\left.\mp\left(T_{0}+e^{\frac{20 \sigma}{3}} V_{0}\right)\right|_{y=0},
$$




$$
\begin{aligned}
\sqrt{\frac{3}{5}}\left|q_{0}\right| \operatorname{chf} & =-\left.\left(T_{0}-\frac{3}{20} e^{\frac{20 \sigma}{3}} \frac{\partial V_{0}}{\partial \sigma}\right)\right|_{y=0}, \\
\left.\frac{\partial V_{0}}{\partial \Phi}\right|_{y=0} & =0 \\
\sqrt{\frac{3}{2}}\left|q_{0}\right| \operatorname{shf} & = \pm\left.\left(T_{1}+e^{\frac{20 \sigma}{3}} V_{1}\right)\right|_{y=y_{1}}, \\
\sqrt{\frac{3}{5}}\left|q_{0}\right| \operatorname{chf} & =+\left.\left(T_{1}-\frac{3}{20} e^{\frac{20 \sigma}{3}} \frac{\partial V_{1}}{\partial \sigma}\right)\right|_{y=y_{1}}, \\
\left.\frac{\partial V_{1}}{\partial \Phi}\right|_{y=y_{1}} & =0
\end{aligned}
$$

where the first sign refers to the $(+,+)$ case, while the second sign refers to the $(-,+)$ case.

The simplest and actually the only solution we were able to find compatible with boundary conditions (66) , for brane potentials of physical interest is, for both $(+,+)$ and $(-,+)$ cases, $C=-\infty$, so that $e^{f}$ is constant

$$
e^{f(y)}=a \quad \text { for } \quad(+,+) \quad ; \quad e^{f(y)}=a^{-1} \quad \text { for } \quad(-,+)
$$

Notice that $\operatorname{ch} f=\sqrt{5 / 3}$ and $\operatorname{sh} f= \pm \sqrt{2 / 3}$ and consequently the boundary conditions take a particularly simple form. Both cases give the same solution for $A$ and $\sigma$

$$
A(y)=\frac{z}{3}|y|+C_{A} \quad, \quad \sigma(y)=\frac{z}{2}|y|+C_{\sigma}
$$

and the metric takes the form:

$$
d s_{5}^{2}=e^{\frac{2}{3} z|y|} \eta_{\mu \nu} d x^{\mu} d x^{\nu}+e^{\frac{20}{3} z|y|+\frac{40}{3} C_{\sigma}} d y^{2}
$$

where $C_{A}$ was absorbed by a rescaling of the $x^{\mu}$ coordinates. By a change of coordinate in $y$, it turns out that (69) is the same as (48), which is the T-dual to the one worked out in [28]. This result reflects the (approximate) supersymmetry of the bulk space at the lowest order in perturbation theory. In this case, combining in a straightforward way the boundary conditions (67) and defining the total brane scalar potentials (including the tension contribution) and their sum

$$
\begin{aligned}
& V_{i, \text { tot }} \equiv V_{i}\left(\phi_{a}\right)+T_{i} e^{-\frac{20 \sigma}{3}} \\
& U(y)=V_{0, \text { tot }} \delta(y)+V_{1, \text { tot }} \delta\left(y-y_{1}\right)
\end{aligned}
$$

we find the local conditions

$$
<V_{i, \text { tot }}>=<V_{S U S Y}>\left.\right|_{y=y_{i}} \quad, \quad<\frac{\partial V_{i, \text { tot }}}{\partial \phi_{a}}>=<\frac{\partial V_{S U S Y}}{\partial \phi_{a}}>\left.\right|_{y=y_{i}},
$$


where $\phi_{a}=\sigma, \Phi$ and where $V_{S U S Y}=q_{i} \exp (-20 \sigma / 3)$ is the BPS (supersymmetric) tension potential. From now on, in order to avoid confusion, we use the notation $<f\left(\phi_{a}\right)>\left.\right|_{y=y_{i}}$ to denote the numerical value of the function $f\left(\phi_{a}\right)$ evaluated by inserting the classical solution $\phi_{a}=\phi_{a}\left(y_{i}\right)$ of the field equations (61) with the boundary conditions (66).

The conditions (71) have an obvious interpretation : since the bulk is supersymmetric to lowest order, branes sources (the potential and its derivatives) should mimic exactly the supersymmetric case. Integrating over the compact coordinate we find the four dimensional integrability conditions

$$
\int_{0}^{y_{1}} d y \sqrt{g_{y y}}<U>=0 \quad, \quad \int_{0}^{y_{1}} d y \sqrt{g_{y y}}<\frac{\partial U}{\partial \phi_{a}}>=0 .
$$

By defining the four dimensional potential

$$
V_{4}=\int_{0}^{y_{1}} d y \sqrt{g_{y y}} U=\left.\left(\sqrt{g_{y y}} V_{0, \text { tot }}\right)\right|_{y=0}+\left.\left(\sqrt{g_{y y}} V_{1, \text { tot }}\right)\right|_{y=y_{1}},
$$

we find the transparent four-dimensional conditions

$$
<V_{4}>=0 \quad, \quad<\frac{\partial V_{4}}{\partial \phi_{a}}>=0,
$$

where in $4 \mathrm{~d}$ the $\langle f>$ symbol has now the standard interpretation of evaluating the function $f$ in the vacuum of the $4 \mathrm{~d}$ theory, obtained by minimizing the $4 \mathrm{~d}$ potential $V_{4}$. The second equation (74) defines the minima of the potential, while the first reminds us that we are searching for a Minkowski solution and therefore the $4 \mathrm{~d}$ cosmological constant is zero in the vacuum.

Since in $y=0$ we have a BPS brane, the simplest and most natural solution is $V_{0}=0$ and this is the case we are considering to start with.

Let us consider concrete examples of scalar potentials, of the form

$$
V_{1}=\alpha_{1} e^{\beta_{1} \sigma}+\alpha_{2} e^{\beta_{2} \sigma} .
$$

The boundary conditions impose the relation:

$$
\alpha_{1}\left(1+\frac{3}{20} \beta_{1}\right)<e^{\beta_{1} \sigma}>\left.\right|_{y=y_{1}}+\alpha_{2}\left(1+\frac{3}{20} \beta_{2}\right)<e^{\beta_{2} \sigma}>\left.\right|_{y=y_{1}}=0 .
$$

In the following we are discussing 2 cases :

(i) $\alpha_{2}=0$. In this case the boundary conditions in $y=y_{1}$ imply:

$$
\beta_{1}=-\frac{20}{3} \quad \text { and } \quad \alpha_{1}=\left|q_{0}\right|-T_{1}<0 .
$$

The total potential becomes: 


$$
V_{1, \text { tot }}=T_{1} e^{-\frac{20}{3} \sigma}+\alpha_{1} e^{-\frac{20}{3} \sigma}=q_{1} e^{-\frac{20}{3} \sigma}
$$

We recover the supersymmetric case, with a BPS brane in $y=y_{1}$ and $y_{1}$ that is not stabilized. Notice that $\left\langle\frac{\partial V_{1, \text { tot }}}{\partial \phi}>=0\right.$ automatically, and therefore all equations of motion and boundary conditions are satisfied in this case. We can now add, in order to stabilize the dilaton, NS-NS and RR fluxes.

(ii) $\beta_{1}=\frac{20}{3}, \beta_{2}=0$. The boundary conditions imply :

$$
\alpha_{1}<e^{\frac{40}{3} \sigma}>\left.\right|_{y=y_{1}}=T_{1}-\left|q_{0}\right|>0 \quad \text { and } \quad \alpha_{2}=-2 \sqrt{\alpha_{1}\left(T_{1}-\left|q_{0}\right|\right)}<0 .
$$

Notice, by using (55) $\alpha_{1}<<1$, that from (179) the volume modulus $\sigma$ is stabilized to a very large value, qualitatively of the order of magnitude given by the naive argument with the (incomplete) scalar potential (156). By inserting into (179) the classical solution ([68) we find

$$
e^{\frac{20 z y_{1}}{3}+\frac{40 C_{\sigma}}{3}}=\frac{T_{1}-q_{1}}{\alpha_{1}}>>1
$$

We see that $y_{1}$ can be stabilized to a moderately large value and therefore it creates no potential phenomenological problems like deviations from the gravitational attraction at macroscopic distances. Actually only a linear combination of $\sigma$ and $y_{1}$ is stabilized by (80). In order to separately stabilize $\sigma$ and $y_{1}$, nontrivial dynamics in $y=0$ seems to be necessary.

The total potential reads now :

$$
V_{1, \text { tot }}=\left(\sqrt{T_{1}-\left|q_{0}\right|} e^{-\frac{10}{3} \sigma}-\sqrt{\alpha_{1}} e^{\frac{10}{3} \sigma}\right)^{2}+q_{1} e^{-\frac{20}{3} \sigma}
$$

By using (79), the positive (squared) term vanishes evaluated as a solution of the classical field equations and the (expectation value of the) scalar potential and its derivative mimic, as is required by (71), the supersymmetric potential. As a consequence, $\left.\left\langle\frac{\partial V_{1, \text { tot }}}{\partial \phi}\right\rangle\right|_{y=y_{1}}=0$ is automatically satisfied, so again a constant dilaton (eventually stabilized by fluxes) is still a solution after adding the induced brane potential. The four dimensional scalar potential in this case is

$$
V_{4}=\left(\sqrt{T_{1}-\left|q_{0}\right|}-\sqrt{\alpha_{1}} e^{\frac{20}{3} \sigma}\right)^{2}
$$

and is positive definite, like in supersymmetric theories. Before adding fluxes, we find therefore results very similar to the no-scale supergravity models [29]: positive definite scalar potential and one flat direction (the dilaton). This is intriguing and is, presumably, related to two facts. First of all, the bulk being almost supersymmetric and coupling to the non-BPS system in $y=y_{1}$, the full effective lagrangian has a non-linearly realized supersymmetry on the nonBPS system. The condition of having static solutions and bulk supersymmetry force the brane scalar potentials to have a form similar to the standard supergravity lagrangian which, in all 
effective string models, is of the no-scale type. Therefore, despite the non-BPS system in $y=y_{1}$, the dynamics responsible for the stabilization of the fields $\sigma$ and $y_{1}$, which describe Kahler moduli in string language, respect constraints very similar to models with spontaneously broken supersymmetry. This point clearly deserves, in our opinion, further and more detailed studies.

We can add, consistently with the boundary conditions (66), induced brane potentials on the $y=0$ brane, in order to stabilize both $\sigma$ and $y_{1}$ moduli. The simplest example doing the job is the positive potential $V_{0}=\left(\gamma_{1} \exp \left(\delta_{1} \sigma\right)+\gamma_{2} \exp \left(\delta_{2} \sigma\right)\right)^{2}$. In this case $\sigma$ is stabilized via the condition $\gamma_{1} \exp \left(\delta_{1} C_{\sigma}\right)+\gamma_{2} \exp \left(\delta_{2} C_{\sigma}\right)=0$ and then $y_{1}$ is stabilized via (80). This example is similar to the racetrack examples of heterotic dilaton stabilization 30. In analogy with the previous example, $V_{0}$ can, via $\gamma_{i}$, depend on the dilaton, which is, however, still not stabilized by the $y=0$ brane dynamics.

\section{2 de Sitter solution}

We are looking for solutions of the form:

$$
\begin{aligned}
d s_{5}^{2} & =e^{2 A(y)+2 C(t)}\left(-d t^{2}+\delta_{i j} d x^{i} d x^{j}\right)+e^{2 B(y)} d y^{2}, \\
F_{5} & =\tilde{f}(t, y) \epsilon_{5}, \quad \sigma=\sigma(y), \quad \Phi=\Phi(y) .
\end{aligned}
$$

As before we can easily determine the solution for the five form from its equation of motion:

$$
\tilde{f}=-q_{0} k_{5}^{2} e^{4 A+B+4 C-\frac{40}{3} \sigma} \epsilon(y)
$$

Fixing the gauge to $B=20 \sigma / 3$ we are left with the equations ${ }^{9}$ :

$$
\begin{aligned}
& -\left(2 \ddot{C}+\dot{C}^{2}\right) e^{-2 A-2 C+\frac{40 \sigma}{3}}+3 A^{\prime \prime}+6 A^{\prime 2}-20 A^{\prime} \sigma^{\prime}+\frac{20}{3} \sigma^{2}+\frac{1}{4}\left(q_{0} k_{5}^{2}\right)^{2}= \\
& =-k_{5}^{2}\left[T_{0} \delta(y)+T_{1} \delta\left(y-y_{1}\right)\right]-k_{5}^{2} e^{\frac{20 \sigma}{3}}\left[V_{0} \delta(y)+V_{1} \delta\left(y-y_{1}\right)\right], \\
& 3 \dot{C}^{2} e^{-2 A-2 C+\frac{40 \sigma}{3}}-3 A^{\prime \prime}-6 A^{\prime 2}+20 A^{\prime} \sigma^{\prime}-\frac{20}{3} \sigma^{\prime 2}-\frac{1}{4}\left(q_{0} k_{5}^{2}\right)^{2}= \\
& =k_{5}^{2}\left[T_{0} \delta(y)+T_{1} \delta\left(y-y_{1}\right)\right]+k_{5}^{2} e^{\frac{20 \sigma}{3}}\left[V_{0} \delta(y)+V_{1} \delta\left(y-y_{1}\right)\right], \\
& -3\left(\ddot{C}+\dot{C}^{2}\right) e^{-2 A-2 C+\frac{40 \sigma}{3}}+6 A^{\prime 2}-\frac{20}{3} \sigma^{\prime 2}+\frac{1}{4}\left(q_{0} k_{5}^{2}\right)^{2}=0, \\
& \sigma^{\prime \prime}+4 A^{\prime} \sigma^{\prime}-\frac{20}{3} \sigma^{\prime 2}+\frac{1}{4}\left(q_{0} k_{5}^{2}\right)^{2}= \\
& =-\frac{k_{5}^{2}}{2}\left[T_{0} \delta(y)+T_{1} \delta\left(y-y_{1}\right)\right]+\frac{3}{40} k_{5}^{2} e^{\frac{20 \sigma}{3}}\left[\delta(y) \frac{\partial V_{0}}{\partial \sigma}+\delta\left(y-y_{1}\right) \frac{\partial V_{1}}{\partial \sigma}\right]
\end{aligned}
$$

\footnotetext{
${ }^{9}$ The notation we use is ${ }^{\prime}=\frac{d}{d y}$ and ${ }^{\circ}=\frac{d}{d t}$.
} 


$$
\Phi^{\prime \prime}+\Phi^{\prime}\left(4 A^{\prime}-\frac{20}{3} \sigma^{\prime}\right)=0
$$

The first two equations determine the function $C(t)$ which, after shifting the origin of time, is given by :

$$
\ddot{C}=\dot{C}^{2} \Rightarrow e^{2 C}=\frac{1}{C_{1}^{2} t^{2}}
$$

and therefore (83) describes a warped $4 \mathrm{~d}$ de Sitter metric.

If the cosmological constant, $\Lambda=C_{1}$, is small we can look for solutions that are small perturbations around the Minkowski solution:

$$
A=A_{0}+\tilde{A} \quad, \quad \sigma=\sigma_{0}+\tilde{\sigma} \quad, \quad \Phi=\Phi_{0}+\tilde{\phi}
$$

where $A_{0}$ and $\sigma_{0}$ are the Minkowski solutions and $\tilde{A}$ and $\tilde{\sigma}$ are small perturbations.

The linearized field equations take the form:

$$
\begin{aligned}
& 3 \tilde{A}^{\prime \prime}+\tilde{A}^{\prime}\left(12 A_{0}^{\prime}-20 \sigma_{0}^{\prime}\right)+\tilde{\sigma}\left(\frac{40}{3} \sigma_{0}^{\prime}-20 A_{0}^{\prime}\right)-3 C_{1}^{2} e^{-2 A_{0}+\frac{40}{3} \sigma_{0}}=0, \\
& \tilde{\sigma}^{\prime \prime}+4 \tilde{A}^{\prime} \sigma_{0}^{\prime}+\tilde{\sigma}^{\prime}\left(4 A_{0}^{\prime}-\frac{40}{3} \sigma_{0}^{\prime}\right)=0 \\
& 12 \tilde{A}^{\prime} A_{0}^{\prime}-\frac{40}{3} \tilde{\sigma}^{\prime} \sigma_{0}^{\prime}-6 C_{1}^{2} e^{-2 A_{0}+\frac{40}{3} \sigma_{0}}=0 \\
& \tilde{\phi}^{\prime \prime}+\tilde{\phi}^{\prime}\left(4 A_{0}-\frac{20}{3} \sigma_{0}\right)=0 .
\end{aligned}
$$

Strictly speaking there are also 4d localized sources in (88). The scalar potentials (55)-(56) inspired by the gaugino condensation are themselves small. The sources in (88) are proportional to them and also to the small perturbations and are therefore quadratically small.

The last two equations allow to determine a first order equation for the variable $\tilde{\sigma}^{\prime}=g$ :

$$
g^{\prime}+4 g\left(A_{0}^{\prime}-\frac{10}{3} \sigma_{0}^{\prime}+\frac{10}{9} \frac{\sigma_{0}^{\prime 2}}{A_{0}^{\prime}}\right)=-2 \frac{\sigma_{0}^{\prime}}{A_{0}^{\prime}} C_{1}^{2} e^{-2 A_{0}+\frac{40}{3} \sigma_{0}} .
$$

Looking for solution of the form $g=f \chi$, with $f$ the solution of the homogeneous equation we find:

$$
f=e^{-4 \int\left(A_{0}^{\prime}-\frac{10}{3} \sigma_{0}^{\prime}+\frac{10}{9} \frac{\sigma_{0}^{\prime 2}}{A_{0}^{\prime}}\right) d y+C_{f}}, \chi=-2 C_{1}^{2} \int \frac{1}{f} \frac{\sigma_{0}^{\prime}}{A_{0}^{\prime}} e^{-2 A_{0}+\frac{40}{3} \sigma_{0}} d y+C_{\chi}
$$

We showed therefore that de Sitter solutions exists, at least in the vicinity of Minkowski one. In order to get a small four-dimensional cosmological constant, we need basically the same fine-tuning as in the Minkowski solutions. 
Using the Minkowski solution found in Section 5.1 the linearized field equations (88) become:

$$
\begin{aligned}
& \tilde{A}^{\prime \prime}-2 z \tilde{A}^{\prime}=C_{1}^{2} e^{6 z y-2 C_{A}+\frac{40}{3} C_{\sigma}}, \\
& \tilde{\sigma}^{\prime \prime}+2 z \tilde{A}^{\prime}-\frac{16}{3} \tilde{\sigma}^{\prime}=0 \\
& 2 z \tilde{A}^{\prime}-\frac{10}{3} \tilde{\sigma}^{\prime}=3 C_{1}^{2} e^{6 z y-2 C_{A}+\frac{40}{3} C_{\sigma}}, \\
& \tilde{\phi}^{\prime \prime}+\tilde{\phi}^{\prime}\left(-2 z y+4 C_{A}-\frac{20}{3} C_{\sigma}\right)=0 .
\end{aligned}
$$

Finally the metric and dilaton read:

$$
\begin{aligned}
& d s_{5}^{2}=e^{\frac{2}{3} z|y|+\frac{C_{0}}{z} e^{2 z|y|}+C_{A \sigma} \frac{C_{1}^{2}}{12 z^{2}} e^{6 z|y|}}\left[\frac{1}{C_{1}^{2} t^{2}}\left(-d t^{2}+\delta_{i j} d x^{i} d x^{j}\right)+\right.
\end{aligned}
$$

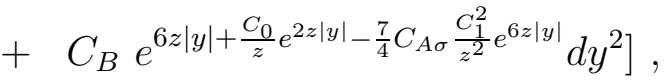

$$
\begin{aligned}
& \Phi=\Phi_{0}+\frac{C}{2 z} e^{2 z|y|}+C_{\phi} .
\end{aligned}
$$

It is likely, but we didn't check it, that in a different range of the parameters there are also 4d anti de Sitter solutions. The main point of all our discussions on nonpertubative dynamics versus moduli stabilization is that, due to the smallness of the nonperturbative scalar potentials, moduli are stabilized at large values by allowing hierarchies to be generated.

\section{Conclusions}

String models with broken supersymmetry are the natural candidates to study cosmology and moduli stabilization. We showed that non-tachyonic orbifold string vacua in various dimensions can be constructed, starting from Scherk-Schwarz compactifications in the closed sector, with the would-be tachyon removed by an appropriate orientifold projection which generates a peculiar $O p_{+}-\overline{O p}_{-}$orientifold plane structure. Including by consistency the open sector, we find $D p-\overline{O p}_{-}$systems which are very similar to the brane-antibrane systems, that were shown by A.Sen [17] to have interesting cosmological applications, but are now tachyon-free, allowing for a clean classical description of their dynamics.

Our non-tachyonic and non-BPS vacua generate simple time-dependent solutions corresponding to (one or some of the) spacetime boundaries moving with a constant velocity in a static bulk, subject classically to a boost identification. Gauge (and Yukawa) couplings on the D3 branes are time-independent at tree-level and acquire a logarithmic time-dependence (51) very similar to that induced by the standard RG equations. For asymptotically free gauge 
groups, which are generically obtained in the non-BPS configurations $D 3-\bar{O}_{-}$of our string vacua, after an exponentially (in fundamental string units) long time (52) the non-BPS branes enter a nonperturbative regime and fix part of the scalar moduli, including the fields describing the size of compact space, but not the (T-dual) dilaton. This exponentially long time can generate hierarchies between the fundamental string scale and the Planck scale. In order to stabilize the internal radii, which are Kahler moduli in string language, we showed that stabilization potential must produce exactly the same sources (vev's of the potentials and their first derivatives) as in the supersymmetric situation. We started the study of the "inverse problem" of determining additional brane potentials compatible with static solutions in the non-BPS vacua. In the particular case of gaugino condensation on the non-BPS boundary, we need to combine the condensate with a constant potential term into a positive definite scalar potential, in close analogy with the dilaton stabilization in the heterotic string [25]. Contrary to the heterotic example, however, in our case the Kahler moduli are fixed, whereas the dilaton remains a flat direction that can subsequently be stabilized by adding appropriate fluxes. After stabilization, the classical solution becomes exactly the same as the supersymmetric one, nonperturbative brane dynamics "repairing" the non-BPS nature of brane sources in order to match the corresponding BPS one.

The exponentially long time period of validity of our cosmological solutions and the subsequent nonperturbative phenomena triggering moduli stabilization raise the hope of possible imprints of string physics in early cosmology. From this perspective, an analysis along the lines of [31] of the class of models we constructed in the present paper would be very interesting and useful to perform.

Acknowledgments. We thanks Jihad Mourad and Augusto Sagnotti for useful discussions. The work of E.D. was supported in part by the RTN European Program HPRN-CT-2000-00148. E.D. is grateful for the warm hospitality at TPI-Minnesota and the Theory Division of CERN at early stages of this work.

\section{Appendix : Characters for $Z_{2} \times Z_{2}$ orbifolds}

Our conventions for writing partition functions and defining moduli for various string surfaces are the ones in the third and the fourth reference in [7]. The level one $\mathrm{SO}(2 \mathrm{n})$ characters are:

$$
\begin{aligned}
O_{2 n}=\frac{\theta_{3}^{n}+\theta_{4}^{n}}{2 \eta^{n}}, & V_{2 n}=\frac{\theta_{3}^{n}-\theta_{4}^{n}}{2 \eta^{n}}, \\
S_{2 n}=\frac{\theta_{2}^{n}+i^{n} \theta_{1}^{n}}{2 \eta^{n}}, & C_{2 n}=\frac{\theta_{2}^{n}-i^{n} \theta_{1}^{n}}{2 \eta^{n}} .
\end{aligned}
$$


The $Z_{2} \times Z_{2}$ torus partition function in the text is written in terms of the following supersymmetric characters [23, 32]:

$$
\begin{aligned}
& \tau_{\text {oo }}=V_{2} \mathrm{O}_{2} \mathrm{O}_{2} \mathrm{O}_{2}+\mathrm{O}_{2} V_{2} V_{2} V_{2}-S_{2} S_{2} S_{2} S_{2}-C_{2} C_{2} C_{2} C_{2} \\
& \tau_{o g}=\mathrm{O}_{2} \mathrm{~V}_{2} \mathrm{O}_{2} \mathrm{O}_{2}+\mathrm{V}_{2} \mathrm{O}_{2} V_{2} V_{2}-\mathrm{C}_{2} \mathrm{C}_{2} \mathrm{~S}_{2} \mathrm{~S}_{2}-\mathrm{S}_{2} \mathrm{~S}_{2} \mathrm{C}_{2} \mathrm{C}_{2} \\
& \tau_{\text {oh }}=\mathrm{O}_{2} \mathrm{O}_{2} \mathrm{O}_{2} \mathrm{~V}_{2}+\mathrm{V}_{2} \mathrm{~V}_{2} \mathrm{~V}_{2} \mathrm{O}_{2}-\mathrm{C}_{2} \mathrm{~S}_{2} \mathrm{~S}_{2} \mathrm{C}_{2}-\mathrm{S}_{2} \mathrm{C}_{2} \mathrm{C}_{2} \mathrm{~S}_{2} \\
& \tau_{\text {of }}=\mathrm{O}_{2} \mathrm{O}_{2} \mathrm{~V}_{2} \mathrm{O}_{2}+\mathrm{V}_{2} \mathrm{~V}_{2} \mathrm{O}_{2} V_{2}-\mathrm{C}_{2} \mathrm{~S}_{2} \mathrm{C}_{2} \mathrm{~S}_{2}-\mathrm{S}_{2} \mathrm{C}_{2} \mathrm{~S}_{2} \mathrm{C}_{2} \\
& \tau_{g o}=V_{2} O_{2} S_{2} C_{2}+O_{2} V_{2} C_{2} S_{2}-S_{2} S_{2} V_{2} O_{2}-C_{2} C_{2} O_{2} V_{2} \\
& \tau_{g g}=\mathrm{O}_{2} V_{2} \mathrm{~S}_{2} \mathrm{C}_{2}+\mathrm{V}_{2} \mathrm{O}_{2} \mathrm{C}_{2} \mathrm{~S}_{2}-\mathrm{S}_{2} \mathrm{~S}_{2} \mathrm{O}_{2} \mathrm{~V}_{2}-\mathrm{C}_{2} \mathrm{C}_{2} \mathrm{~V}_{2} \mathrm{O}_{2} \\
& \tau_{g h}=\mathrm{O}_{2} \mathrm{O}_{2} \mathrm{~S}_{2} \mathrm{~S}_{2}+\mathrm{V}_{2} \mathrm{~V}_{2} \mathrm{C}_{2} \mathrm{C}_{2}-\mathrm{C}_{2} \mathrm{~S}_{2} \mathrm{~V}_{2} \mathrm{~V}_{2}-\mathrm{S}_{2} \mathrm{C}_{2} \mathrm{O}_{2} \mathrm{O}_{2} \\
& \tau_{g f}=\mathrm{O}_{2} \mathrm{O}_{2} \mathrm{C}_{2} \mathrm{C}_{2}+\mathrm{V}_{2} \mathrm{~V}_{2} \mathrm{~S}_{2} \mathrm{~S}_{2}-\mathrm{S}_{2} \mathrm{C}_{2} \mathrm{~V}_{2} \mathrm{~V}_{2}-\mathrm{C}_{2} \mathrm{~S}_{2} \mathrm{O}_{2} \mathrm{O}_{2} \\
& \tau_{h o}=V_{2} S_{2} C_{2} O_{2}+O_{2} C_{2} S_{2} V_{2}-C_{2} O_{2} V_{2} C_{2}-S_{2} V_{2} O_{2} S_{2} \\
& \tau_{h g}=\mathrm{O}_{2} \mathrm{C}_{2} \mathrm{C}_{2} \mathrm{O}_{2}+\mathrm{V}_{2} \mathrm{~S}_{2} \mathrm{~S}_{2} \mathrm{~V}_{2}-\mathrm{C}_{2} \mathrm{O}_{2} \mathrm{O}_{2} \mathrm{~S}_{2}-\mathrm{S}_{2} \mathrm{~V}_{2} \mathrm{~V}_{2} \mathrm{C}_{2} \\
& \tau_{h h}=\mathrm{O}_{2} \mathrm{~S}_{2} \mathrm{C}_{2} \mathrm{~V}_{2}+\mathrm{V}_{2} \mathrm{C}_{2} \mathrm{~S}_{2} \mathrm{O}_{2}-\mathrm{S}_{2} \mathrm{O}_{2} \mathrm{~V}_{2} \mathrm{~S}_{2}-\mathrm{C}_{2} \mathrm{~V}_{2} \mathrm{O}_{2} \mathrm{C}_{2} \\
& \tau_{h f}=\mathrm{O}_{2} S_{2} S_{2} O_{2}+V_{2} C_{2} C_{2} V_{2}-C_{2} V_{2} V_{2} S_{2}-S_{2} O_{2} O_{2} C_{2} \\
& \tau_{f o}=V_{2} S_{2} O_{2} C_{2}+O_{2} C_{2} V_{2} S_{2}-S_{2} V_{2} S_{2} O_{2}-C_{2} O_{2} C_{2} V_{2} \\
& \tau_{f g}=\mathrm{O}_{2} \mathrm{C}_{2} \mathrm{O}_{2} \mathrm{C}_{2}+\mathrm{V}_{2} \mathrm{~S}_{2} \mathrm{~V}_{2} \mathrm{~S}_{2}-\mathrm{C}_{2} \mathrm{O}_{2} \mathrm{~S}_{2} \mathrm{O}_{2}-\mathrm{S}_{2} \mathrm{~V}_{2} \mathrm{C}_{2} V_{2} \\
& \tau_{f h}=\mathrm{O}_{2} \mathrm{~S}_{2} \mathrm{O}_{2} \mathrm{~S}_{2}+\mathrm{V}_{2} \mathrm{C}_{2} \mathrm{~V}_{2} \mathrm{C}_{2}-\mathrm{C}_{2} \mathrm{~V}_{2} \mathrm{~S}_{2} \mathrm{~V}_{2}-\mathrm{S}_{2} \mathrm{O}_{2} \mathrm{C}_{2} \mathrm{O}_{2} \\
& \tau_{f f}=\mathrm{O}_{2} \mathrm{~S}_{2} V_{2} C_{2}+\mathrm{V}_{2} \mathrm{C}_{2} \mathrm{O}_{2} \mathrm{~S}_{2}-\mathrm{C}_{2} V_{2} \mathrm{C}_{2} \mathrm{O}_{2}-\mathrm{S}_{2} \mathrm{O}_{2} \mathrm{~S}_{2} V_{2}
\end{aligned}
$$

and nonsupersymmetric combinations [22]

$$
\begin{aligned}
\tau_{o o}^{\prime} & =V_{2} O_{2} O_{2} O_{2}+O_{2} V_{2} V_{2} V_{2}-C_{2} S_{2} S_{2} C_{2}-S_{2} C_{2} C_{2} S_{2} \\
\tau_{o g}^{\prime} & =O_{2} V_{2} O_{2} O_{2}+V_{2} O_{2} V_{2} V_{2}-C_{2} S_{2} C_{2} S_{2}-S_{2} C_{2} S_{2} C_{2} \\
\tau_{o h}^{\prime} & =O_{2} O_{2} O_{2} V_{2}+V_{2} V_{2} V_{2} O_{2}-S_{2} S_{2} S_{2} S_{2}-C_{2} C_{2} C_{2} C_{2} \\
\tau_{o f}^{\prime} & =O_{2} O_{2} V_{2} O_{2}+V_{2} V_{2} O_{2} V_{2}-C_{2} C_{2} S_{2} S_{2}-S_{2} S_{2} C_{2} C_{2} \\
\tau_{g o}^{\prime} & =O_{2} O_{2} S_{2} C_{2}+V_{2} V_{2} C_{2} S_{2}-S_{2} S_{2} V_{2} V_{2}-C_{2} C_{2} O_{2} O_{2} \\
\tau_{g g}^{\prime} & =O_{2} O_{2} C_{2} S_{2}+V_{2} V_{2} S_{2} C_{2}-S_{2} S_{2} O_{2} O_{2}-C_{2} C_{2} V_{2} V_{2} \\
\tau_{g h}^{\prime} & =V_{2} O_{2} S_{2} S_{2}+O_{2} V_{2} C_{2} C_{2}-S_{2} C_{2} O_{2} V_{2}-C_{2} S_{2} V_{2} O_{2} \\
\tau_{g f}^{\prime} & =O_{2} V_{2} S_{2} S_{2}+V_{2} O_{2} C_{2} C_{2}-C_{2} S_{2} O_{2} V_{2}-S_{2} C_{2} V_{2} O_{2} \\
\tau_{h o}^{\prime} & =V_{2} S_{2} C_{2} O_{2}+O_{2} C_{2} S_{2} V_{2}-S_{2} O_{2} V_{2} S_{2}-C_{2} V_{2} O_{2} C_{2} \\
\tau_{h g}^{\prime} & =O_{2} C_{2} C_{2} O_{2}+V_{2} S_{2} S_{2} V_{2}-C_{2} V_{2} V_{2} S_{2}-S_{2} O_{2} O_{2} C_{2} \\
\tau_{h h}^{\prime} & =O_{2} S_{2} C_{2} V_{2}+V_{2} C_{2} S_{2} O_{2}-C_{2} O_{2} V_{2} C_{2}-S_{2} V_{2} O_{2} S_{2} \\
\tau_{h f}^{\prime} & =O_{2} S_{2} S_{2} O_{2}+V_{2} C_{2} C_{2} V_{2}-C_{2} O_{2} O_{2} S_{2}-S_{2} V_{2} V_{2} C_{2} \\
\tau_{f o}^{\prime} & =O_{2} S_{2} O_{2} C_{2}+V_{2} C_{2} V_{2} S_{2}-S_{2} V_{2} S_{2} V_{2}-C_{2} O_{2} C_{2} O_{2}
\end{aligned}
$$




$$
\begin{aligned}
\tau_{f g}^{\prime} & =O_{2} S_{2} V_{2} S_{2}+V_{2} C_{2} O_{2} C_{2}-C_{2} O_{2} S_{2} V_{2}-S_{2} V_{2} C_{2} O_{2} \\
\tau_{f h}^{\prime} & =V_{2} S_{2} O_{2} S_{2}+O_{2} C_{2} V_{2} C_{2}-S_{2} O_{2} C_{2} V_{2}-C_{2} V_{2} S_{2} O_{2} \\
\tau_{f f}^{\prime} & =O_{2} C_{2} O_{2} S_{2}+V_{2} S_{2} V_{2} C_{2}-S_{2} O_{2} S_{2} O_{2}-C_{2} V_{2} C_{2} V_{2} \\
\sigma_{o o} & =O_{2} O_{2} O_{2} O_{2}+V_{2} V_{2} V_{2} V_{2}-C_{2} S_{2} S_{2} S_{2}-S_{2} C_{2} C_{2} C_{2} \\
\sigma_{o g} & =O_{2} O_{2} V_{2} V_{2}+V_{2} V_{2} O_{2} O_{2}-S_{2} C_{2} S_{2} S_{2}-C_{2} S_{2} C_{2} C_{2} \\
\sigma_{o h} & =O_{2} V_{2} V_{2} O_{2}+V_{2} O_{2} O_{2} V_{2}-S_{2} S_{2} S_{2} C_{2}-C_{2} C_{2} C_{2} S_{2} \\
\sigma_{o f} & =O_{2} V_{2} O_{2} V_{2}+V_{2} O_{2} V_{2} O_{2}-C_{2} C_{2} S_{2} C_{2}-S_{2} S_{2} C_{2} S_{2} \\
\sigma_{h o} & =O_{2} S_{2} C_{2} O_{2}+V_{2} C_{2} S_{2} V_{2}-S_{2} O_{2} V_{2} C_{2}-C_{2} V_{2} O_{2} S_{2} \\
\sigma_{h g} & =O_{2} S_{2} S_{2} V_{2}+V_{2} C_{2} C_{2} O_{2}-S_{2} O_{2} O_{2} S_{2}-C_{2} V_{2} V_{2} C_{2} \\
\sigma_{h h} & =O_{2} C_{2} S_{2} O_{2}+V_{2} S_{2} C_{2} V_{2}-S_{2} V_{2} O_{2} C_{2}-C_{2} O_{2} V_{2} S_{2} \\
\sigma_{h f} & =O_{2} C_{2} C_{2} V_{2}+V_{2} S_{2} S_{2} O_{2}-C_{2} O_{2} O_{2} C_{2}-S_{2} V_{2} V_{2} S_{2}
\end{aligned}
$$

We use the notation $(i=o, g, h, f)$ :

$$
\begin{array}{ll}
T_{i o}=\tau_{i o}+\tau_{i g}+\tau_{i h}+\tau_{i f}, & T_{i g}=\tau_{i o}+\tau_{i g}-\tau_{i h}-\tau_{i f}, \\
T_{i h}=\tau_{i o}-\tau_{i g}+\tau_{i h}-\tau_{i f}, & T_{i f}=\tau_{i o}-\tau_{i g}-\tau_{i h}+\tau_{i f},
\end{array}
$$

and likewise for the $\tau^{\prime}$ 's $\left(T_{i j}^{\prime}\right)$ and the $\sigma$ 's $\left(S_{i j}\right)$; a superscript "F" or "B" for the T's will denote the Fermionic or Bosonic part of the characters. In our convention, $T_{i j}^{B \pm F}=T_{i j}^{B} \pm T_{i j}^{F}$, etc. We also used the definitions

$$
\begin{array}{rlrl}
\Lambda_{m, 2 n} & =\sum_{m, n} \frac{1+(-1)^{n}}{2} \Lambda_{m, n}, & & \Lambda_{m, 2 n+1}=\sum_{m, n} \frac{1-(-1)^{n}}{2} \Lambda_{m, n}, \\
\Lambda_{m+1 / 2,2 n} & =\sum_{m, n} \frac{1+(-1)^{n}}{2} \Lambda_{m+\frac{1}{2}, n} \quad, & \Lambda_{m+1 / 2,2 n+1}=\sum_{m, n} \frac{1-(-1)^{n}}{2} \Lambda_{m+\frac{1}{2}, n},
\end{array}
$$

where $\Lambda_{m, n}$ denotes the standard $(1,1)$ momentum and winding lattice and

$$
P_{m+a}(\tau) \equiv \sum_{m} q^{\frac{\pi \alpha^{\prime}(m+a)^{2}}{R^{2}}} \quad, \quad W_{n+b}(\tau) \equiv \sum_{n} q^{\frac{\pi}{4 \alpha^{\prime}}(n+b)^{2} R^{2}}
$$

\section{References}

[1] R. Rohm, Nucl. Phys. B237 (1984) 553; C. Kounnas and M. Porrati, Nucl. Phys. B 310 (1988) 355 ; S. Ferrara, C. Kounnas, M. Porrati and F. Zwirner, Nucl. Phys. B318 (1989) 75; I. Antoniadis, Phys. Lett. B 246 (1990) 377 ; E. Kiritsis and C. Kounnas, Nucl. Phys. B503 (1997) 117 hep-th/9703059; for recent work and extensive list of references, see for example C. A. Scrucca and M. Serone, JHEP 0110, 017 (2001) arXiv:hep-th/0107159; D. M. Ghilencea, H. P. Nilles and S. Stieberger, New J. Phys. 4, 15 (2002) arXiv:hep-th/0108183. 
[2] C. Bachas, arXiv:hep-th/9503030 ; M. Berkooz, M. R. Douglas and R. G. Leigh, Nucl. Phys. B 480 (1996) 265 arXiv:hep-th/9606139 ; R. Blumenhagen, L. Goerlich, B. Kors and D. Lust, JHEP 0010 (2000) 006 arXiv:hep-th/0007024 ; C. Angelantonj, I. Antoniadis, E. Dudas and A. Sagnotti, Phys. Lett. B 489 (2000) 223 arXiv:hep-th/0007090; G. Aldazabal, S. Franco, L. E. Ibanez, R. Rabadan and A. M. Uranga, J. Math. Phys. 42 (2001) 3103 arXiv:hep-th/0011073.

[3] S. Sugimoto, Prog. Theor. Phys. 102 (1999) 685 hep-th/9905159 ; I. Antoniadis, E. Dudas and A. Sagnotti, Phys. Lett. B 464 (1999) 38 hep-th/9908023; C. Angelantonj, Nucl. Phys. B 566, 126 (2000) arXiv:hep-th/9908064; G. Aldazabal and A. M. Uranga, JHEP 9910 (1999) 024 hep-th/9908072; G. Aldazabal, L. E. Ibanez and F. Quevedo, JHEP 0001, 031 (2000) arXiv:hep-th/9909172; C. Angelantonj, R. Blumenhagen and M. R. Gaberdiel, Nucl. Phys. B 589, 545 (2000) arXiv:hep-th/0006033.

[4] L. J. Dixon and J. A. Harvey, Nucl. Phys. B 274 (1986) 93 ; . Alvarez-Gaume, P. Ginsparg, G. W. Moore and C. Vafa, Phys. Lett. B 171 (1986) 155 ; N. Seiberg and E. Witten, Nucl. Phys. B 276 (1986) 272.

[5] A. Sagnotti, arXiv:hep-th/9509080 Nucl. Phys. Proc. Suppl. 56B, 332 (1997) arXiv:hep-th/9702093 ; C. Angelantonj, Phys. Lett. B 444, 309 (1998) arXiv:hep-th/9810214.

[6] A. Sagnotti, in: Cargese '87, Non-Perturbative Quantum Field Theory, eds. G. Mack et al. (Pergamon Press, Oxford, 1988) p. 521; M. Bianchi and A. Sagnotti, Phys. Lett. B247 (1990) 517, Nucl. Phys. B361 (1991) 519; G. Pradisi and A. Sagnotti, Phys. Lett. B216 (1989) 59; M. Bianchi, G. Pradisi and A. Sagnotti, Nucl. Phys. B376 (1992) 365. P. Horava, Nucl. Phys. B 327, 461 (1989). J. Dai, R. G. Leigh and J. Polchinski, Mod. Phys. Lett. A 4, 2073 (1989).

[7] J. Polchinski, "TASI lectures on D-branes," arXiv:hep-th/9611050 A. Dabholkar, arXiv:hep-th/9804208. E. Dudas, Class. Quant. Grav. 17 (2000) R41 arXiv:hep-ph/0006190 ; C. Angelantonj and A. Sagnotti, "Open strings," arXiv:hep-th/0204089.

[8] J. D. Blum and K. R. Dienes, Nucl. Phys. B 516 (1998) 83 arXiv:hep-th/9707160 ; Nucl. Phys. B 520 (1998) 93 arXiv:hep-th/9708016.

[9] I. Antoniadis, E. Dudas and A. Sagnotti, Nucl. Phys. B544 (1999) 469 hep-th/9807011; I. Antoniadis, G. D'Appollonio, E. Dudas and A. Sagnotti, Nucl. Phys. B553 (1999) 133 hep-th/9812118; R. Blumenhagen and L. Gorlich, Nucl. Phys. B 551, 601 (1999) arXiv:hep-th/9812158; C. Angelantonj, I. Antoniadis and K. Forger, Nucl. Phys. B 555, 116 (1999) arXiv:hep-th/9904092 ; C. A. Scrucca, M. Serone and M. Trapletti, Nucl. 
Phys. B 635 (2002) 33 arXiv:hep-th/0203190; C. Angelantonj and I. Antoniadis, Nucl. Phys. B 676 (2004) 129 arXiv:hep-th/0307254.

[10] E. Dudas and J. Mourad, Nucl. Phys. B 598 (2001) 189 arXiv:hep-th/0010179.

[11] E. Dudas, J. Mourad and C. Timirgaziu, Nucl. Phys. B 660, 3 (2003) arXiv:hep-th/0209176.

[12] E. Dudas, J. Mourad and C. Timirgaziu, arXiv:hep-th/0309057.

[13] W. D. Goldberger and M. B. Wise, Phys. Rev. Lett. 83 (1999) 4922 arXiv:hep-ph/9907447.

[14] S. B. Giddings, S. Kachru and J. Polchinski, Phys. Rev. D 66 (2002) 106006 arXiv:hep-th/0105097 ; S. Kachru, M. B. Schulz and S. Trivedi, JHEP 0310 (2003) 007 arXiv:hep-th/0201028 ; A. R. Frey and J. Polchinski, Phys. Rev. D 65 (2002) 126009 arXiv:hep-th/0201029.

[15] J. Khoury, B. A. Ovrut, N. Seiberg, P. J. Steinhardt and N. Turok, Phys. Rev. D 65 (2002) 086007 arXiv:hep-th/0108187 ; N. Seiberg, arXiv:hep-th/0201039 V. Balasubramanian, S. F. Hassan, E. Keski-Vakkuri and A. Naqvi, arXiv:hep-th/0202187; L. Cornalba and M. S. Costa, arXiv:hep-th/0203031; N. A. Nekrasov, arXiv:hep-th/0203112; L. Cornalba, M. S. Costa and C. Kounnas, Nucl. Phys. B 637 (2002) 378 arXiv:hep-th/0204261; B. Pioline and M. Berkooz, JCAP 0311, 007 (2003) arXiv:hep-th/0307280.

[16] G. T. Horowitz and A. R. Steif, Phys. Lett. B 258, 91 (1991); G. T. Horowitz and A. R. Steif, Phys. Rev. D 42, 1950 (1990); H. Liu, G. Moore and N. Seiberg, JHEP 0206 (2002) 045 arXiv:hep-th/0204168 ; arXiv:hep-th/0206182 J. Simon, JHEP 0206, 001 (2002) arXiv:hep-th/0203201; M. Fabinger and J. McGreevy, arXiv:hep-th/0206196; G. T. Horowitz and J. Polchinski, arXiv:hep-th/0206228.

[17] A. Sen, JHEP 0204, 048 (2002) arXiv:hep-th/0203211, JHEP 0207, 065 (2002) arXiv:hep-th/0203265, Mod. Phys. Lett. A 17, 1797 (2002) arXiv:hep-th/0204143, JHEP 0210, 003 (2002) arXiv:hep-th/0207105.

[18] G. Pradisi, arXiv:hep-th/0310154 M. Larosa and G. Pradisi, Nucl. Phys. B 667 (2003) 261 arXiv:hep-th/0305224; E. Dudas, J. Mourad and G. Pradisi, unpublished, 2002.

[19] C. Angelantonj and R. Blumenhagen, Phys. Lett. B 473 (2000) 86 arXiv:hep-th/9911190.

[20] A. Sagnotti, Phys. Lett. B 294, 196 (1992) arXiv:hep-th/9210127. 
[21] E. Dudas and J. Mourad, Phys. Lett. B 514, 173 (2001) arXiv:hep-th/0012071; J. H. Schwarz and E. Witten, JHEP 0103 (2001) 032 arXiv:hep-th/0103099; G. Pradisi and F. Riccioni, arXiv:hep-th/0107090; I. Antoniadis, K. Benakli and A. Laugier, Nucl. Phys. B 631, 3 (2002) arXiv:hep-th/0111209; M. Klein, arXiv:hep-th/0205300.

[22] A. L. Cotrone, Mod. Phys. Lett. A 14, 2487 (1999) arXiv:hep-th/9909116.

[23] M. Bianchi, Ph.D. thesis, preprint ROM2F-92/13 ; A. Sagnotti, arXiv:hep-th/9302099.

[24] C. Angelantonj, I. Antoniadis, G. D'Appollonio, E. Dudas and A. Sagnotti, Nucl. Phys. B $\mathbf{5 7 2}(2000) 36$ arXiv:hep-th/9911081.

[25] J. P. Derendinger, L. E. Ibanez and H. P. Nilles, Phys. Lett. B 155 (1985) 65 ; M. Dine, R. Rohm, N. Seiberg and E. Witten, Phys. Lett. B 156, 55 (1985).

[26] R. Blumenhagen, D. Lust and T. R. Taylor, Nucl. Phys. B 663, 319 (2003) arXiv:hep-th/0303016 ; J. F. G. Cascales and A. M. Uranga, JHEP 0305 (2003) 011 arXiv:hep-th/0303024.

[27] S. Gukov, C. Vafa and E. Witten, Nucl. Phys. B 584 (2000) 69 [Erratum-ibid. B 608 (2001) 477] arXiv:hep-th/9906070 ; T. R. Taylor and C. Vafa, Phys. Lett. B 474 (2000) 130 arXiv:hep-th/9912152.

[28] J. Polchinski and E. Witten, Nucl. Phys. B 460 (1996) 525 arXiv:hep-th/9510169.

[29] E. Cremmer, S. Ferrara, C. Kounnas and D. V. Nanopoulos, Phys. Lett. B 133, 61 (1983); J. R. Ellis, C. Kounnas and D. V. Nanopoulos, Nucl. Phys. B 241, 406 (1984).

[30] N. V. Krasnikov, Phys. Lett. B 193 (1987) 37 ; J. A. Casas, Z. Lalak, C. Munoz and G. G. Ross, Nucl. Phys. B 347, 243 (1990) ; B. de Carlos, J. A. Casas and C. Munoz, Nucl. Phys. B 399, 623 (1993) arXiv:hep-th/9204012.

[31] R. Easther, B. R. Greene, W. H. Kinney and G. Shiu, Phys. Rev. D 64 (2001) 103502 arXiv:hep-th/0104102; N. Kaloper, M. Kleban, A. E. Lawrence and S. Shenker, Phys. Rev. D 66, 123510 (2002) arXiv:hep-th/0201158.

[32] I. Antoniadis, G. D’Appollonio, E. Dudas and A. Sagnotti, Nucl. Phys. B 565 (2000) 123 arXiv:hep-th/9907184. 